\title{
Chemical Warfare Agent Simulants in Gamble's Fluid: Is the Fluid Toxic? Can It Be Made Safer by Inclusion of Solid Nanocrystalline Metal Oxides?
}

\author{
Dennis Karote, ${ }^{1}$ Brandon Walker, ${ }^{1}$ Huaien Dai, ${ }^{2}$ Ramaswamy Krishnamoorthi, ${ }^{2}$ \\ Janis Voo, ${ }^{1}$ and Shyamala Rajagopalan ${ }^{1}$ \\ ${ }^{1}$ Research \& Development Group, NanoScale Corporation, 1310 Research Park Drive, Manhattan, KS 66502, USA \\ ${ }^{2}$ Department of Biochemistry, Kansas State University, Manhattan, KS 66506, USA
}

Correspondence should be addressed to Shyamala Rajagopalan; srajagopalan@nanoscalecorp.com

Received 11 June 2012; Accepted 9 October 2012

Academic Editor: Meehir Palit

Copyright (C) 2013 Dennis Karote et al. This is an open access article distributed under the Creative Commons Attribution License, which permits unrestricted use, distribution, and reproduction in any medium, provided the original work is properly cited.

\begin{abstract}
The reactions of chemical warfare agent simulants, 2-chloroethyl ethyl sulfide (2-CEES) and di- $i$-propyl fluoro phosphate (DFP), in fluids have been investigated. Data analyses confirm the major degradation pathway to be hydrolysis of 2-CEES to 2-hydroxyethyl ethyl sulfide, along with minor self-condensation products. Among the three fluids examined, 2-CEES degradation was the fastest in Gamble's fluid during a $96 \mathrm{~h}$ period. Upon addition of Exceptional Hazard Attenuation Materials (EHAMs) to 2-CEES containing Gamble's fluid, degradation was generally improved during the first $24 \mathrm{~h}$ period. The $96 \mathrm{~h}$ outcome was similar for fluid samples with or without EHAM 2 and EHAM 4. EHAM 1-added fluid contained only one degradation product, 2-nitroethyl ethyl sulfide. DFP degradation was the slowest in Gamble's fluid, but was enhanced by the addition of EHAMs. FTIR and solid state ${ }^{31} \mathrm{P}$ NMR confirm the destructive adsorption of 2-CEES and DFP by the EHAMs. The results collectively demonstrate that 2-CEES and DFP decompose to various extents in Gamble's fluid over a $96 \mathrm{~h}$ period but the fluid still contains a considerable amount of intact simulant. EHAM 1 appears to be promising for 2-CEES and DFP mitigation while EHAM 2 and EHAM 4 work well for early on concentration reduction of 2-CEES and DFP.
\end{abstract}

\section{Introduction}

Use of chemical and biological warfare agents (CWAs and BWAs) in conventional or terrorism related incidents is not new [1-3]. Despite the longtime use of CWAs, very little is understood about the possibilities of secondary contamination of persons who handle fatalities from these incidents. This lack of understanding and dearth of reliable data makes designing protective systems and processes for dealing with contaminated remains extremely challenging. In fact, fatality management has always been a highly sensitive issue [4]. The various operational, political, and religious constraints associated with the proper search, recovery, identification, and disposition of remains pose a complex problem. A catastrophic event will produce appreciable contaminated casualties that will require very specific approaches to contaminated remains processing and sound decisions have to be quickly made concerning proper handling of the remains.

The handling and transport of contaminated human remains is an important consideration of both combative and humanitarian military actions. Current protocol has remained largely unchanged for decades, although significant advances in decontamination and incident response have been made. Handlers of contaminated and potentially contaminated human remains face significant biological and chemical threats $[5,6]$. Contamination may arise from a deliberate enemy action, such as an Improvised Explosive Device (IED) which also unleashes a toxic gas or hazardous chemical or direct exposure to CWAs, or it may be incidental due to poor sanitation and looser environmental standards 
found in some foreign nations. While the causes of contamination may differ, the end result is the same. There is a need for a system to provide for the safe transportation of contaminated remains from the point of fatality to a Mortuary Affairs Decontamination Collection Point (MADCP) and, following processing and release, on to the Continental United States (CONUS). This system must be sufficiently robust to permit the transportation of remains via multiple means (i.e., helicopter, airplane, boat, or wheeled transport) while being sufficiently versatile to contain and mitigate a variety of contaminant types.

In recognition of this need, NanoScale Corporation (NanoScale), in collaboration with Kappler, Inc., has recently produced a human remains pouch which was specifically designed to allow for safe handling and transport of contaminated human remains (http://www.nanoscalecorp.com/, http://www.kappler.com/home/). This contaminated human remains pouch (CHRP) has been shown to dramatically reduce or eliminate the permeation of multiple toxic industrial chemicals (TICs), as well as CWAs [7]. However, the CHRP, as well as other protective devices, could be made even more effective if more were known about the fate of CWAs on human remains. NanoScale has produced many extremely effective formulations for decontamination, including their flagship product, FAST-ACT ${ }^{\circledR}$, which is capable of mitigating hazards arising from multiple toxic chemicals in different chemical classes. Biologically active formulations able to kill viruses, bacteria, and spores have also been produced. The main focus of NanoScale's business has been decontamination; therefore, NanoScale is keenly interested in exploring the question "What is the outcome of postmortem interaction of CWAs with physiologically active body fluids?"

The objective of this study was thus twofold: (1) to develop highly efficient, dually active (against CWAs and BWAs) solid decontaminants based on nanocrystalline metal oxides, and (2) to develop reliable analytical methods and generate data to quantify the residual CWA simulants and degradation products that may be present in contaminated human remains. Specifically, we have investigated the degradation of CWA simulants in Gamble's fluid, a synthetic equivalent of physiological fluid, with and without the inclusion of novel dually active solid decontaminants. Over the past several years, NanoScale has developed a series of highly reactive nanocrystalline metal oxides and their derivatives, which have been shown to be effective in destructive adsorption of a number of toxic compounds, including the CWAs, soman (GD), VX, and mustard (HD) [8-11]. Destructive adsorption by nanocrystalline materials leads to chemical conversion of the CWAs into far less toxic compounds, thereby permanently eliminating the threat. The unique properties of these mesoporous adsorbents are due to their high surface areas, unusual morphologies and high surface concentrations of reactive defect sites. In oxides such as $\mathrm{MgO}$, $\mathrm{Al}_{2} \mathrm{O}_{3}$, and $\mathrm{TiO}_{2}$, nerve agents are adsorbed by means of hydrogen-bonds between the surface hydroxyl groups and the $\mathrm{P}=\mathrm{O}$ moieties, followed by sequential removal of the functional groups by hydrolysis. This leaves behind a stable phosphate attached to the surface (Scheme 1). Similarly, HD undergoes decomposition by elimination/hydrolysis induced by the metal oxide materials. Despite the intense interest in exploiting the novel properties of these metal oxides as dry materials, studies directed towards their performance in a fluid environment are scarce.

Herein, efforts to evaluate the fate of CWA simulants di-i-propyl fluoro phosphonate (DFP; simulant for nerve agent) and 2-chloroethyl ethyl sulfide (2-CEES; simulant for mustard) in a variety of fluids with and without the inclusion of solid decontaminants are reported. Analytical techniques involving gas chromatography (GC), Fourier transform infrared (FTIR) spectroscopy, and nuclear magnetic resonance (NMR) spectroscopy were used. Results presented herein should aid in developing a preliminary assessment of potential human health and safety risks that may arise from exposure to residual levels of agents present in the contaminated remains.

\section{Materials and Methods}

2.1. Materials. Deuterium oxide (99.9\% D) was purchased from Cambridge Isotope Laboratories, Inc. (Andover, MA). Deuterated chloroform $(99.8 \%$ D) was purchased from ACROS Organics (Morris Plains, NJ, USA). 2-CEES (98\% pure), ethyl vinyl sulfide (EVS, 96\% pure), and DFP were purchased from Aldrich (St. Louis, MO, USA). 2-(ethylthio) ethanol (96\% pure), also known as hydroxylethyl ethyl sulfide (HEES), was purchased from Alfa Aesar (Ward Hill, MA, USA). Isopropanol ( $>99 \%$ pure) was purchased from Barton Solvent, Inc. (Des Moines, IA, USA). Sodium chloride (ACS grade), ammonium chloride (ACS grade), sodium citrate tribasic (ACS grade), sodium bicarbonate (ACS grade), glycine (ACS grade), calcium chloride (ACS grade), and sodium phosphate dibasic (ACS grade) were purchased from Sigma-Aldrich (St. Louis, MO, USA). Titanium (IV) oxide (certified grade, $\mathrm{FS}-\mathrm{TiO}_{2}$ ) and diethyl ether (lab grade) were purchased from Fisher (Pittsburg, PA, USA). NanoActive $\mathrm{TiO}_{2}\left(\mathrm{NA}-\mathrm{TiO}_{2}\right)$ and NanoActive ${ }^{\circledR} \mathrm{CaO}(\mathrm{NA}-\mathrm{CaO})$ were manufactured by NanoScale Corporation (Manhattan, KS, USA). All chemicals were used as received with no further purification.

Three silver based metal oxide materials were prepared and assessed for their CWA simulant decontamination ability. These materials are referred to as Exceptional Hazard Attenuation Materials (EHAMs), designated EHAM 1, EHAM 2, and EHAM 4. EHAM $1\left(\mathrm{SSA}=264 \mathrm{~m}^{2} / \mathrm{g}\right)$ was based on Ag-impregnated NanoActive $\mathrm{TiO}_{2}\left([\mathrm{Ag}] \mathrm{NA}-\mathrm{TiO}_{2}\right)$. EHAM $2\left(\mathrm{SSA}=213 \mathrm{~m}^{2} / \mathrm{g}\right)$ contains $[\mathrm{Ag}] \mathrm{NA}-\mathrm{TiO}_{2}$ and [Ag]NA-CaO. EHAM $4\left(\mathrm{SSA}=88 \mathrm{~m}^{2} / \mathrm{g}\right)$ contains [Ag]FS$\mathrm{TiO}_{2}$, NA-CaO and NA-TiO .

Saline solutions were prepared at a concentration of $0.9 \%$ (w/w). Gamble's fluid was prepared according to the published literature [12]. The composition of Gamble's fluid is sodium chloride $(6.78 \mathrm{~g} / \mathrm{L})$; ammonium chloride $(0.53 \mathrm{~g} / \mathrm{L})$; sodium bicarbonate $(2.27 \mathrm{~g} / \mathrm{L})$; glycine $(0.45 \mathrm{~g} / \mathrm{L})$; L-Cysteine $(0.12 \mathrm{~g} / \mathrm{L})$; sodium citrate tribasic $(0.06 \mathrm{~g} / \mathrm{L})$; calcium chloride $(0.02 \mathrm{~g} / \mathrm{L})$; sodium phosphate dibasic $(0.17 \mathrm{~g} / \mathrm{L})$. For nonNMR sample preparations, Elix water was used. For saline 

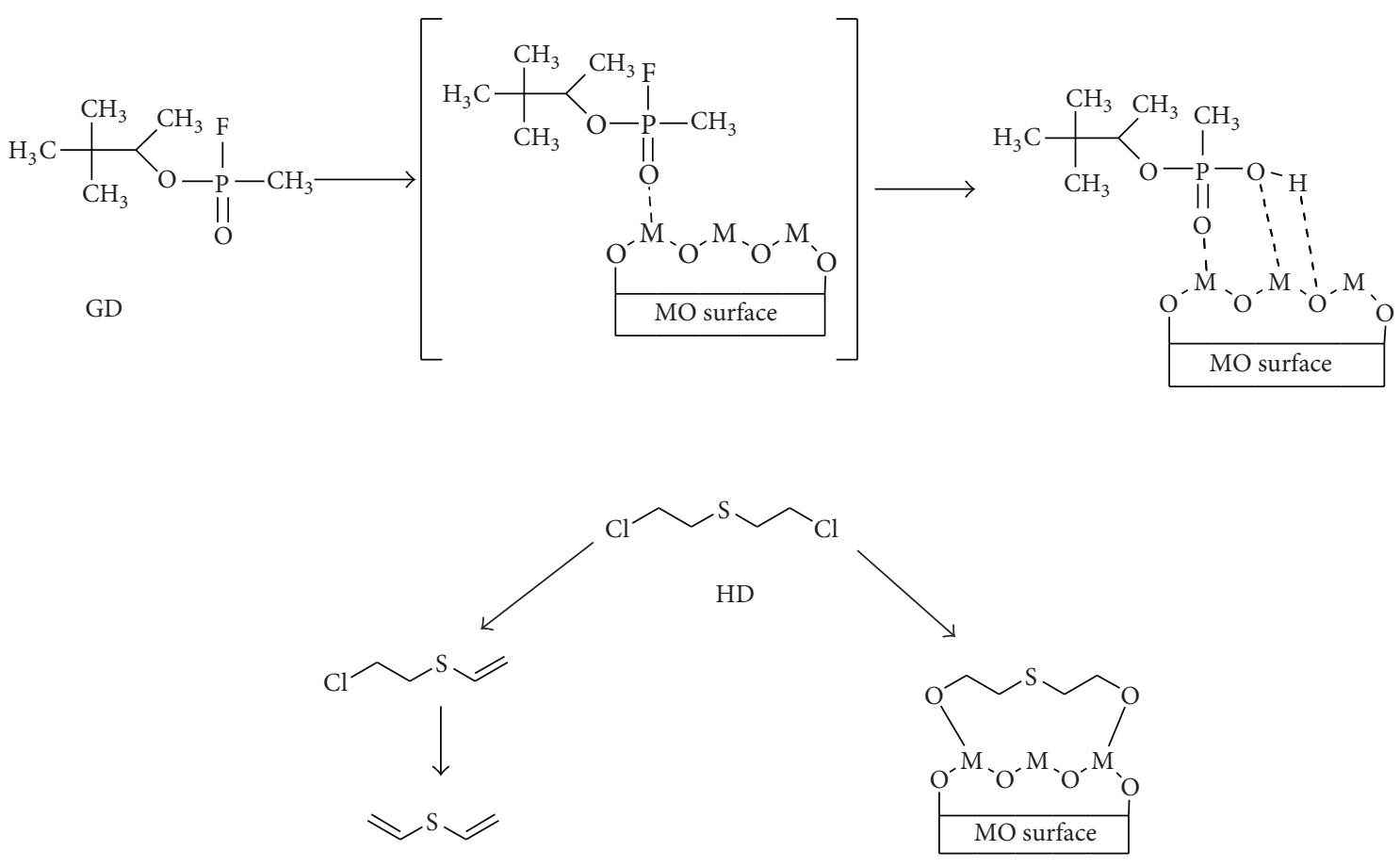

SCHeme 1: Decomposition of nerve/blister agents on metal oxide surfaces.

and Gamble's fluid used in NMR experiments, $\mathrm{D}_{2} \mathrm{O}$ was used in place of water.

2.2. $p H$ Analysis. The fates of the individual simulants, 2CEES and DFP, in fluids were investigated by measuring $\mathrm{pH}$ of the solutions as a function of time, as likely reaction products were expected to increase the acidity of a given simulant solution. Thus, $\mathrm{pH}$ measurements were made on aqueous, $0.9 \%$ saline, and Gamble's fluid solutions of 2-CEES and DFP with a Fisher Scientific, accumet AB15 pH meter. In a representative procedure, $20 \mu \mathrm{L}$ of simulant was added to $2 \mathrm{~mL}$ of fluid (water, saline, or Gamble's fluid), stirred, and the $\mathrm{pH}$ was measured after $10 \mathrm{~min}, 1 \mathrm{~h}, 96 \mathrm{~h}$, and 8 days of the preparation. The $\mathrm{pH}$ of the fluid was measured prior to the addition of simulant for comparison.

2.3. GC Analysis. GC analysis was conducted to determine the fate of the simulant in fluids with and without any EHAM present. A Hewlett Packard 5890 Series II GC equipped with a Hewlett Packard 5972 Mass Selective Detector and a HP-5MS capillary column was used for sample analysis. Since 2-CEES and HEES coeluted on this column, quantitation of 2-CEES and HEES was performed using a Hewlett Packard 5890 Series II GC equipped with a Flame Photometric Detector utilizing a 5\% SE-30 ON CW-HP 80/100 packed column. In a typical experiment, $5 \mu \mathrm{L}$ simulant (2-CEES or DFP) was added to $0.5 \mathrm{~mL}$ fluid (water, saline, or Gamble's fluid), vortexed, and left for 1, 24, or $96 \mathrm{~h}$. The solution was extracted with $1 \mathrm{~mL}$ ethyl ether and analyzed by GC-MS and GC-FPD. Control samples containing $5 \mu \mathrm{L}$ simulant in $1 \mathrm{~mL}$ ether were used to calculate the amount of simulant remaining. A second set of GC experiments was carried out to determine the fate of the simulants in the presence of EHAMs. For this study, $20 \mu \mathrm{L}$ simulant was added to $2 \mathrm{~mL}$ of Gamble's fluid, along with $600 \mathrm{mg}$ of EHAM. Samples were vortexed briefly and allowed to sit at room temperature for a contact time of 1,24 , or $96 \mathrm{~h}$. Subsequently, a $0.5 \mathrm{~mL}$ sample was removed using a syringe filter. This fluid was extracted with $1 \mathrm{~mL}$ ethyl ether and analyzed by GC-MS and GC-FPD. Control samples containing $20 \mu \mathrm{L}$ simulant (2-CEES or DFP) in $2 \mathrm{~mL}$ ether were used to calculate the relative amounts of the remaining simulant and any by-products produced.

2.4. FTIR Analysis. FTIR spectra of the powder samples were recorded in the absorbance mode using a Thermo Scientific Nicolet 6700 spectrophotometer. The FTIR unit was equipped with a HeNe laser source, and an MCT detector cooled with liquid nitrogen. A Praying Mantis Diffuse Reflectance Infrared Fourier Transform spectroscopy (DRIFTS) unit with purging nitrogen was used for the sample analysis at ambient temperature. All spectra were collected by coadding 1032 scans at a resolution of $4 \mathrm{~cm}^{-1}$. The spectra were recorded from 4000 to $500 \mathrm{~cm}^{-1}$.

Due to the instrumentation detection limit, a higher agent concentration was needed to perform the DRIFTS experiments. As a result, all of the FTIR related samples were prepared in duplicate with a 2-CEES to sorbent (EHAM 1, EHAM 2, or EHAM 4) ratio of $1: 10$ (wt:wt). In a typical reaction, $9.3 \mu \mathrm{L}$ 2-CEES was placed in a GC vial, followed by the addition of $100 \mathrm{mg}$ of the test sorbent. The GC vial was capped, the mixture was allowed to react under ambient conditions for 1,24 , and $96 \mathrm{~h}$, and the IR spectra of the samples were acquired. Background subtraction of the acquired spectra was performed using the appropriate 
sorbent spectrum to obtain the final FTIR spectrum of the reacted sorbent.

2.5. NMR Analysis. Solution NMR experiments were performed at Kansas State University, Manhattan, KS, USA, using a11.75 $\mathrm{T}\left(500 \mathrm{MHz}\right.$ for $\left.{ }^{1} \mathrm{H}\right)$ Varian UNITYplus spectrometer (Varian, Palo Alto, CA, USA). NMR samples were prepared by adding $20 \mu \mathrm{L}$ of simulant (2-CEES or DFP) to $2 \mathrm{~mL}$ solvent $\left(\mathrm{CDCl}_{3}, \mathrm{D}_{2} \mathrm{O}\right.$, saline, or Gamble's fluid) and then vortexing briefly to mix. For samples involving EHAM sorbents, $20 \mu \mathrm{L}$ of agent was added to $2 \mathrm{~mL}$ solvent, quickly mixed by vortexing, followed by the addition of $600 \mathrm{mg}$ of sorbent. The mixture was stirred for the desired contact time $(1,24$, or $96 \mathrm{~h})$ before being filtered for NMR analysis.

${ }^{1} \mathrm{H}$ NMR spectra were acquired at room temperature, using a spectral width of $6010 \mathrm{~Hz}$ over $8 \mathrm{~K}$ data points. A relaxation delay of $1 \mathrm{~s}$ was employed for a 45 degree flip angle. Four scans were coadded for each sample and Fourier-transformed with a line-broadening factor of $0.5 \mathrm{~Hz}$ to improve the $\mathrm{S} / \mathrm{N}$ ratio. Chemical shifts were referenced against the solvent peak (7.24 ppm for $\mathrm{CDCl}_{3}$ and $4.8 \mathrm{ppm}$ for HOD). Relative amounts of various products formed were estimated from corresponding methyl peak intensities expressed as percentages of the total intensity of all methyl peaks in a spectrum. This permitted comparison of NMR data as a function of time not only for a given sample, but also between samples to obtain semiquantitative estimates of reaction rates.

Solid state proton-decoupled ${ }^{31} \mathrm{P}$ NMR spectra were obtained at Iowa State University, Ames, IA, USA, with a Bruker AVANCE II $14.1 \mathrm{~T}\left(242.9 \mathrm{MHz}\right.$ for $\left.{ }^{31} \mathrm{P}\right)$ equipped with a Bruker PH MASDVT600WB H/X/Y probe under the condition of magic angle spinning (MAS) at a rate of $5-10 \mathrm{KHz}$, using a spectral width of $73529 \mathrm{~Hz}$ over $4 \mathrm{k}$ time domain points; a relaxation delay of $10 \mathrm{~s}$ was used for a 90 degree pulse; 32 scans were collected for each sample, a linebroadening factor of $20 \mathrm{~Hz}$ was employed to improve the $\mathrm{S} / \mathrm{N}$ ratio. Chemical shifts are reported relative to the external standard, phosphoric acid, which is assigned a value of $0 \mathrm{ppm}$. Typically, $20 \mu \mathrm{L}$ of agent was added to $600 \mathrm{mg}$ of sorbent and vortexed briefly to mix. After the desired contact time, the sample was homogenized thoroughly with a spatula and packed into a Bruker $4 \mathrm{~mm}$ zirconia MAS rotor for data collection.

\section{Results}

3.1. $p H$ Analysis. $\mathrm{pH}$ values of individual solutions of 2CEES and DFP in water, $0.9 \%$ saline solution, and Gamble's fluid were measured after $10 \mathrm{~min}, 1 \mathrm{~h}, 96 \mathrm{~h}$, and 8 days of preparation. The results (Figure 1) indicate hydrolysis and degradation of these compounds into products that increase acidity.

3.2. GC Analysis. Agent-contacted fluid and sorbent were extracted with ethyl ether after 1,24 , and $96 \mathrm{~h}$ of preparation and analyzed by gas chromatography. Results were analyzed in terms of all the possible 2-CEES and DFP degradation products, as summarized in Schemes 2 and 3, respectively. The assignments were confirmed by GC-MS fragmentation pattern as well as NMR data and by comparison with data from authentic samples. The fluids and sorbent reacted with 2-CEES and generated several by-products within $1 \mathrm{~h}$ and the reaction(s) continued up to $96 \mathrm{~h}$. The relative amounts (normalized against an external control) of remaining 2CEES and by-products are reported in Tables 1 and 2.

Similarly, the fluids and sorbent reacted with DFP within the first hour of contact and the amount of DFP present continued to decrease during the $96 \mathrm{~h}$ period (Figures 2(a) and 2(b)). Because of nonvolatility of phosphonic acid-based reaction products, only the relative amounts of the remaining DFP at various time points are reported. Among the examined fluids (Figure 2(a)), degradation was the slowest in Gamble's. The results presented in Figure 2(b) show that EHAMs 2 and 4 are especially efficient for early on reduction of DFP.

3.3. FTIR Studies. The FTIR spectrum of neat 2-CEES is shown in Figure 3, and the bands characteristic of $\mathrm{C}-\mathrm{Cl}$ functionality are marked $\left(1215 \mathrm{~cm}^{-1} \mathrm{Cl}-\mathrm{C}-\mathrm{H}_{2}\right.$ scissor and $695 \mathrm{~cm}^{-1} \mathrm{C}-\mathrm{Cl}$ stretch). The FTIR spectrum for the reaction of dry EHAM 1 and 2-CEES (Figure 4) remained identical for the 1,24, and $96 \mathrm{~h}$ time periods. Even though several bands were observed in all of the spectra, the bands due to $\mathrm{C}-\mathrm{Cl}$ functionality were not present. Two new bands (not observed in neat 2-CEES) at $1640 \mathrm{~cm}^{-1}$ and $1280 \mathrm{~cm}^{-1}$ were present in all spectra.

In contrast to EHAM 1, EHAM 2-treated samples (Figure 5) exhibited time sensitive spectral variations. The variations were specifically obvious in the $3700-3500$ and $1550-1400 \mathrm{~cm}^{-1}$ regions. The IR bands due to $\mathrm{Cl}-\mathrm{C}-\mathrm{CH}_{2}$ scissor and $\mathrm{C}-\mathrm{Cl}$ stretch were absent. Overall, the spectral bands for EHAM 4-contacted samples remained relatively identical for the three time periods (Figure 6). As mentioned earlier for EHAM 2, the bands assigned to the $\mathrm{C}-\mathrm{Cl}$ functionality were not present.

\subsection{NMR Analysis}

3.4.1. Solution ${ }^{1} H$ NMR Studies. An assigned ${ }^{1} \mathrm{H}$ NMR spectrum of 2-CEES in $\mathrm{CDCl}_{3}$ is shown in Figure 7. When 2-CEES was examined in an aqueous environment, several new peaks were observed. The ${ }^{1} \mathrm{H}$ NMR spectra of 2-CEES in $\mathrm{D}_{2} \mathrm{O}$, saline, and Gamble's fluid were very similar. The spectra of 2-CEES in Gamble's fluid, examined after 1, 24, and $96 \mathrm{~h}$ of preparation, are shown in Figure 8. The spectra become complex because of formation of at least three degradation products (Figure 8), as deduced from the appearance of four groups of methyl peaks. Changes in relative intensities of these methyl peaks allowed us to monitor the time course of disappearance of 2-CEES and formation of new reaction products (Table 3 ).

${ }^{1} \mathrm{H}$ NMR spectra of 2-CEES in fluids in the presence of EHAMs are shown in Figures 9(a)-9(c). The spectra for 2CEES in Gamble's fluid treated with EHAM 1 indicate the formation of two products initially, with the amount of the 


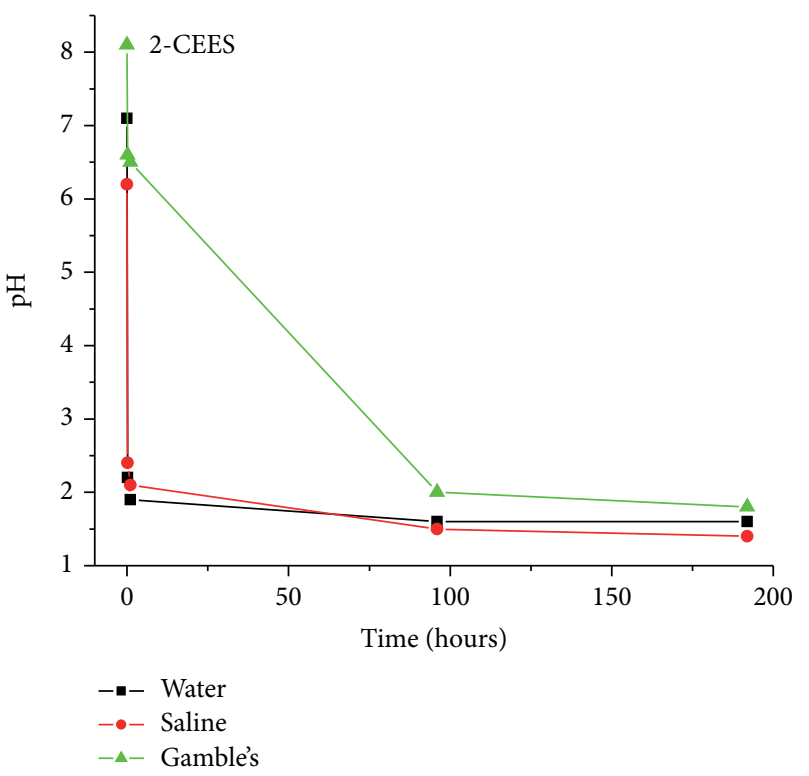

(a)

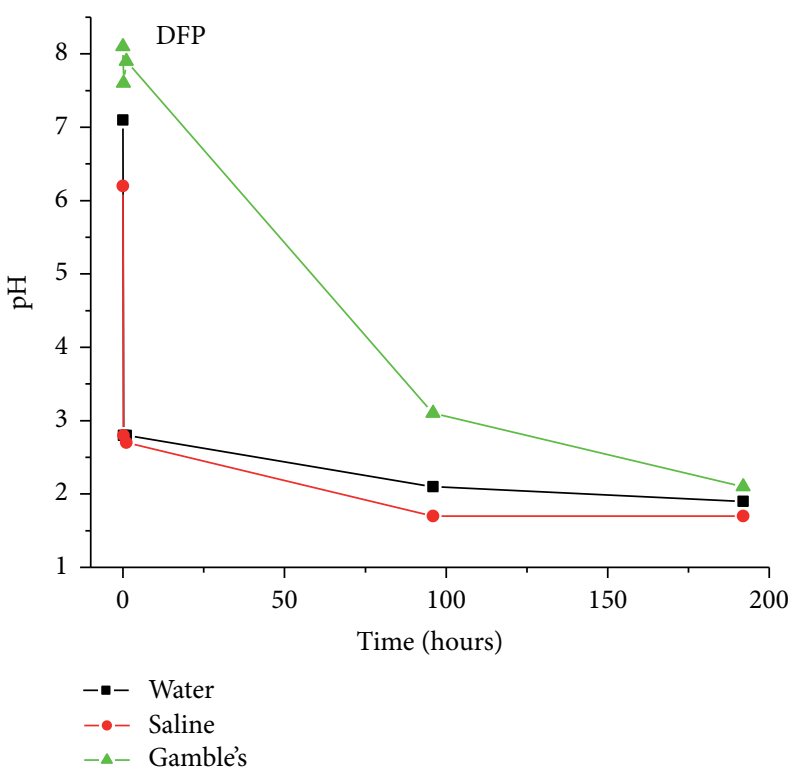

(b)

FIGURE 1: $\mathrm{pH}$ of simulant-containing fluids at various time periods.

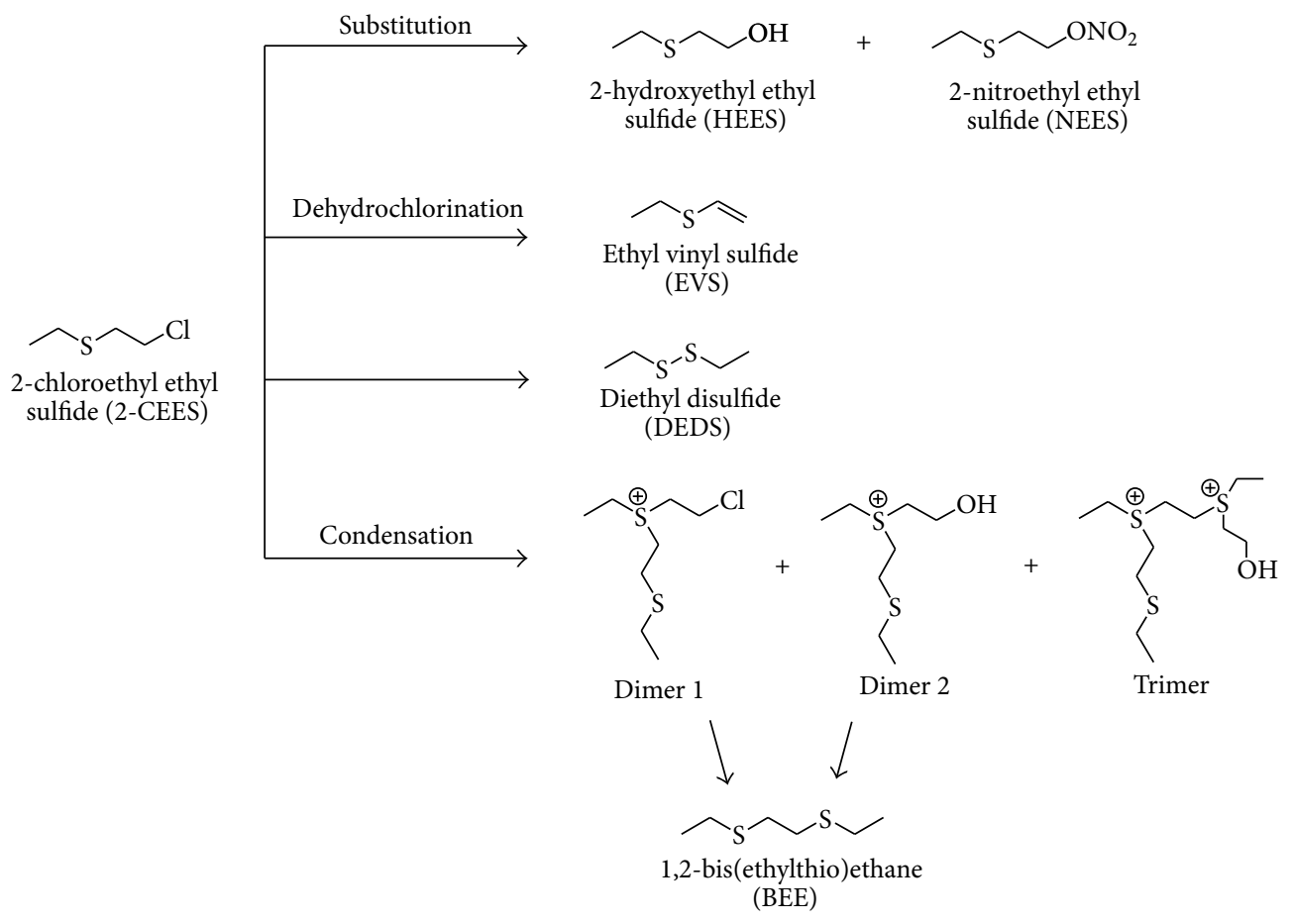

SCHeme 2: Degradation products of 2-CEES.

minor product decreasing over the $96 \mathrm{~h}$ period, suggesting further degradation of this compound. The peak patterns suggest that both products have the basic structure similar to HEES and CEES. The identity of the major product is inferred to be nitroethyl ethyl sulfide (NEES) on the basis of GC-MS fragmentation pattern. In contrast, both EHAM 2 and EHAM 4 yielded multiple products, when reacted with 2-CEES in
Gamble's fluid (Figures 9(b) and 9(c), resp.), as seen in the upfield methyl region (1.1-1.4 ppm). Table 4 summarizes the relative intensity data for all the products present in the fluid.

${ }^{1} \mathrm{H}$ NMR spectra of DFP in $\mathrm{CDCl}_{3}, \mathrm{D}_{2} \mathrm{O}$, saline, and Gamble's fluid are shown in Figures 10(a)-10(d), respectively. At least, four different products containing isopropyl group were seen in addition to intact DFP after $96 \mathrm{~h}$ in Gamble's 


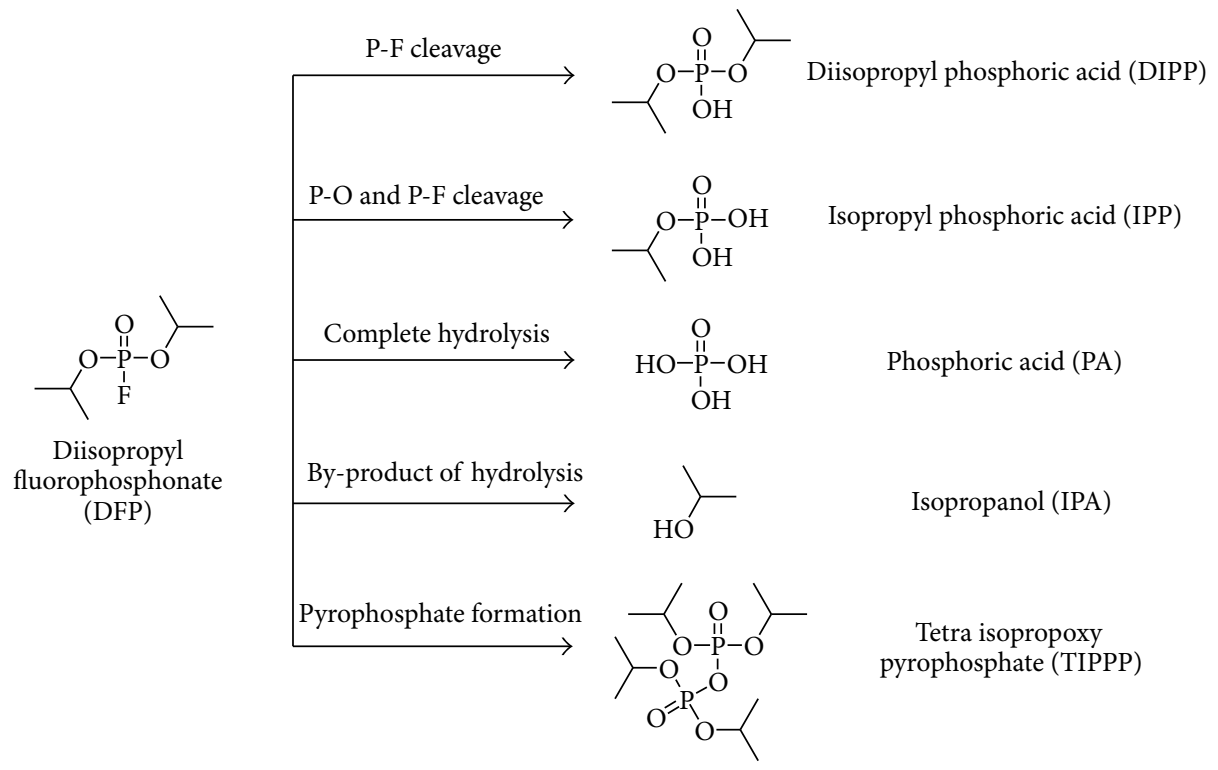

SCHEME 3: Degradation products of DFP.

TABLE 1: Relative (to external 2-CEES control) amounts of extractable products from various fluids.

\begin{tabular}{|c|c|c|c|c|c|c|c|c|}
\hline Fluid & Contact time (hours) & 2-CEES & HEES & DEDS & NEES & EVS & $\mathrm{BEE}$ & Trimer \\
\hline \multirow{3}{*}{ Water } & 1 & 2.1 & 13 & $<024$ & \multirow{3}{*}{$\mathrm{BDL}$} & $<006$ & \multirow{3}{*}{$\leq 0.15$} & \multirow{3}{*}{$\leq 0.20$} \\
\hline & 24 & 0.91 & 42 & -0.24 & & -0.00 & & \\
\hline & 96 & 1.1 & 84 & BDL & & $\mathrm{BDL}$ & & \\
\hline \multirow{3}{*}{ Saline } & 1 & 5.6 & 26 & \multirow{2}{*}{$\leq 0.14$} & \multirow{3}{*}{$\mathrm{BDL}$} & 5.6 & \multirow{3}{*}{$\leq 0.22$} & \multirow{3}{*}{$\leq 0.16$} \\
\hline & 24 & 1.9 & 37 & & & $\mathrm{BDL}$ & & \\
\hline & 96 & 3.5 & 73 & $\mathrm{BDL}$ & & DDL & & \\
\hline \multirow{3}{*}{ Gamble's } & 1 & 2.7 & 14 & $<0.32$ & \multirow{3}{*}{$\mathrm{BDL}$} & \multirow{3}{*}{$\mathrm{BDL}$} & \multirow{3}{*}{$\leq 0.13$} & \multirow{3}{*}{$\leq 0.17$} \\
\hline & 24 & 1.9 & 28 & $\leq 0.32$ & & & & \\
\hline & 96 & 7.1 & 78 & $\mathrm{BDL}$ & & & & \\
\hline
\end{tabular}

TABLE 2: Relative (to external 2-CEES control) amounts of extractable products from Gamble's fluid in the presence of EHAM.

\begin{tabular}{|c|c|c|c|c|c|c|c|c|}
\hline EHAM & Contact time (hours) & 2-CEES & HEES & DEDS & NEES & EVS & $\mathrm{BEE}$ & Trimer \\
\hline \multirow{3}{*}{1} & 1 & 28 & 1.1 & \multirow{3}{*}{$\leq 1.3$} & \multirow{3}{*}{$\leq 0.08$} & \multirow{3}{*}{$\mathrm{BDL}$} & \multirow{3}{*}{$\leq 0.0007$} & \multirow{3}{*}{$\leq 0.79$} \\
\hline & 24 & \multirow{2}{*}{$\leq 0.70$} & 1.4 & & & & & \\
\hline & 96 & & 1.5 & & & & & \\
\hline \multirow{3}{*}{2} & 1 & \multirow{3}{*}{$\leq 0.05$} & 28 & \multirow{3}{*}{ BDL } & \multirow{3}{*}{$\mathrm{BDL}$} & \multirow{3}{*}{$\leq 1.3$} & \multirow{3}{*}{$\leq 1.9$} & \multirow{3}{*}{$\leq 0.09$} \\
\hline & 24 & & 18 & & & & & \\
\hline & 96 & & 60 & & & & & \\
\hline \multirow{3}{*}{4} & 1 & \multirow{3}{*}{$\leq 0.05$} & 34 & \multirow{3}{*}{ BDL } & \multirow{3}{*}{$\mathrm{BDL}$} & \multirow{3}{*}{$\leq 0.47$} & \multirow{3}{*}{$\leq 0.40$} & \multirow{3}{*}{$\leq 0.09$} \\
\hline & 24 & & 62 & & & & & \\
\hline & 96 & & 64 & & & & & \\
\hline
\end{tabular}

TABLE 3: Comparison of methyl-peak intensities of 2-CEES reaction products.

\begin{tabular}{|c|c|c|c|c|c|c|c|c|c|}
\hline \multirow{3}{*}{ Peak } & \multicolumn{9}{|c|}{ Relative intensity percentage } \\
\hline & \multicolumn{3}{|c|}{$\mathrm{D}_{2} \mathrm{O}$} & \multicolumn{3}{|c|}{ Saline } & \multicolumn{3}{|c|}{ Gamble's } \\
\hline & $1 \mathrm{~h}$ & $24 \mathrm{~h}$ & $96 \mathrm{~h}$ & $1 \mathrm{~h}$ & $24 \mathrm{~h}$ & $96 \mathrm{~h}$ & $1 \mathrm{~h}$ & $24 \mathrm{~h}$ & $96 \mathrm{~h}$ \\
\hline $\mathrm{b}$ & 13 & 11 & 28 & 16 & 10 & 34 & 25 & 34 & 61 \\
\hline $\mathrm{a}$ & 40 & 45 & 34 & 35 & 43 & 35 & 33 & 33 & 18 \\
\hline c & 34 & 40 & 38 & 37 & 42 & 41 & 28 & 33 & 22 \\
\hline d & 13 & 4 & 0 & 13 & 5 & 0 & 15 & 0 & 0 \\
\hline Assignment & \multicolumn{3}{|c|}{ HEES (b) } & \multicolumn{3}{|c|}{ 2-CEES (a) } & \multicolumn{3}{|c|}{ Dimer and/or trimer, (c and d) } \\
\hline
\end{tabular}




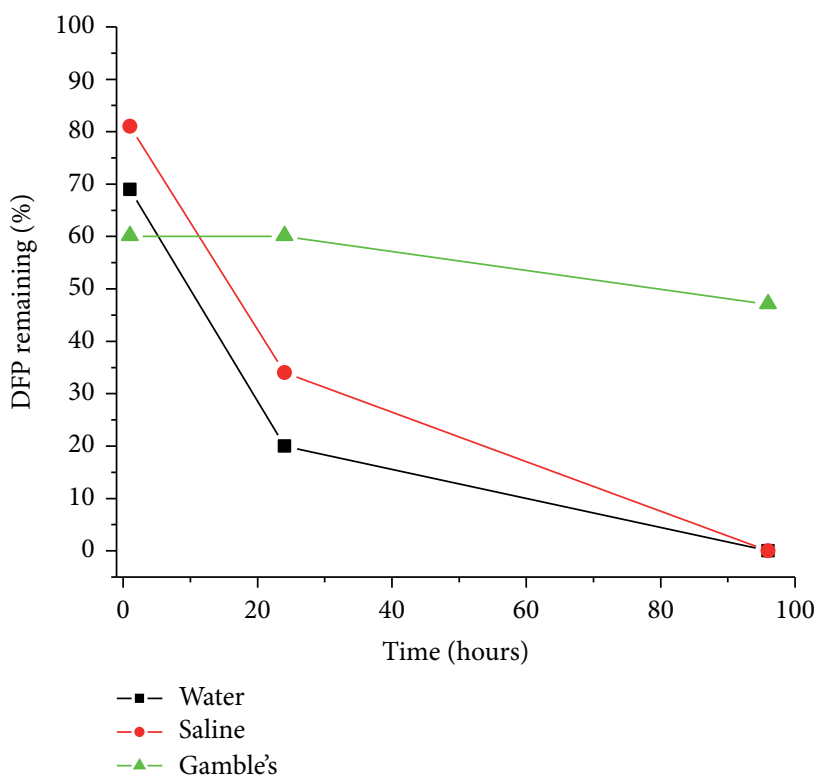

(a)

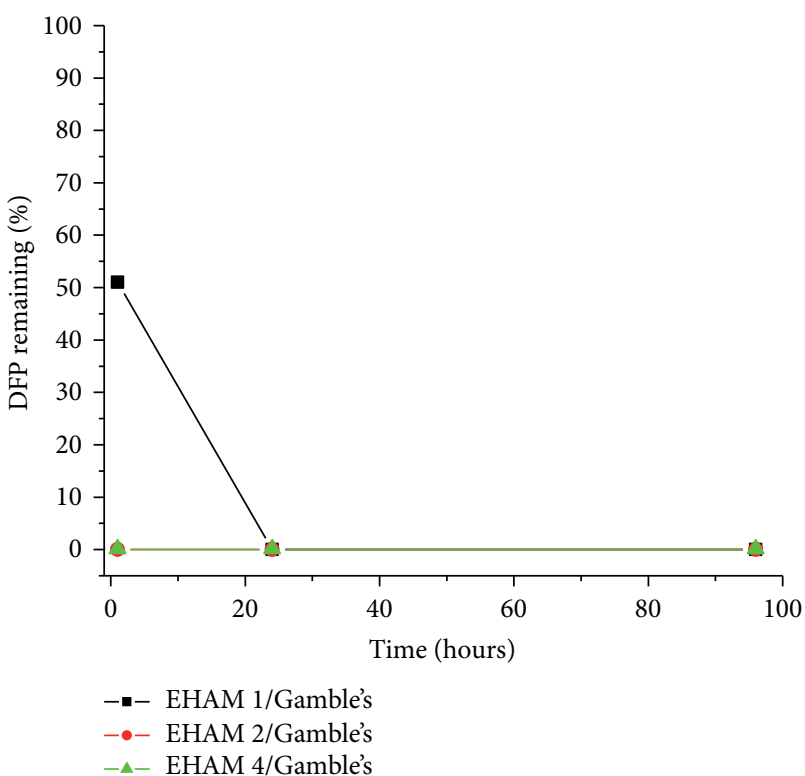

(b)

Figure 2: (a) Fate of DFP in fluids, (b) fate of DFP in Gamble's fluid with EHAMs.

TABLE 4: Comparison of methyl-peak intensities of 2-CEES reaction products.

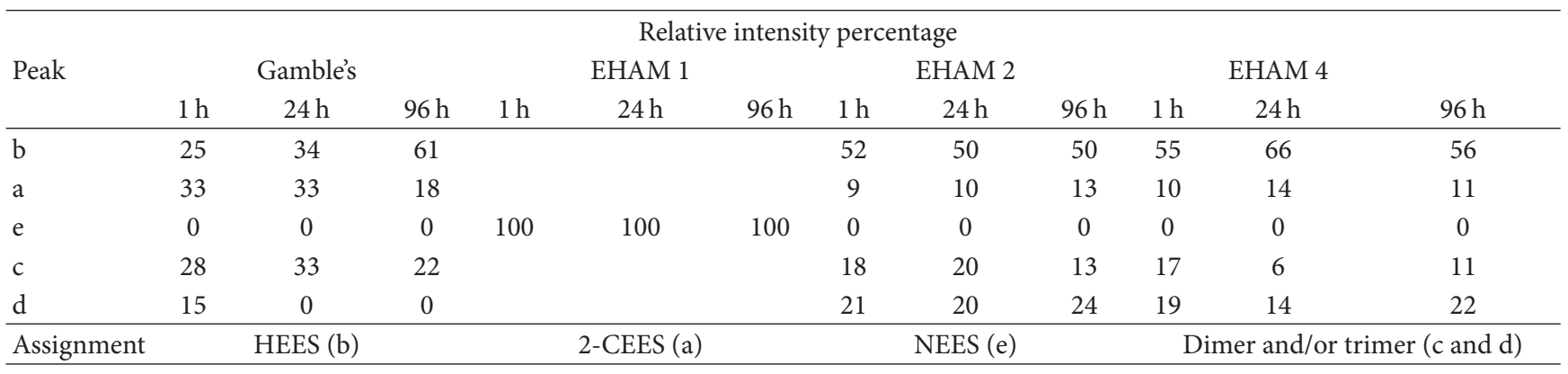

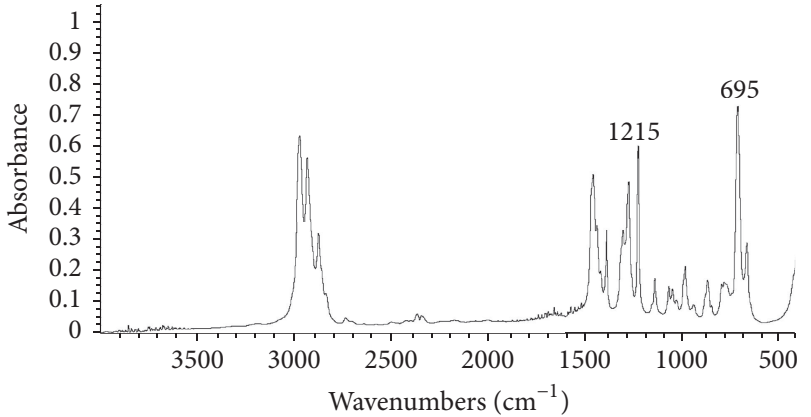

Figure 3: FTIR spectrum of 2-CEES.

fluid (Scheme 3). In sharp contrast, complete/near complete degradation was observed in $\mathrm{D}_{2} \mathrm{O}$ and saline, respectively. All compounds containing an isopropyl group yielded a doublet around $1-1.3 \mathrm{ppm}$ and a multiplet around $3-5 \mathrm{ppm}$. One of these compounds was identified as IPA on the basis of a reference spectrum obtained of that compound in $\mathrm{D}_{2} \mathrm{O}$.

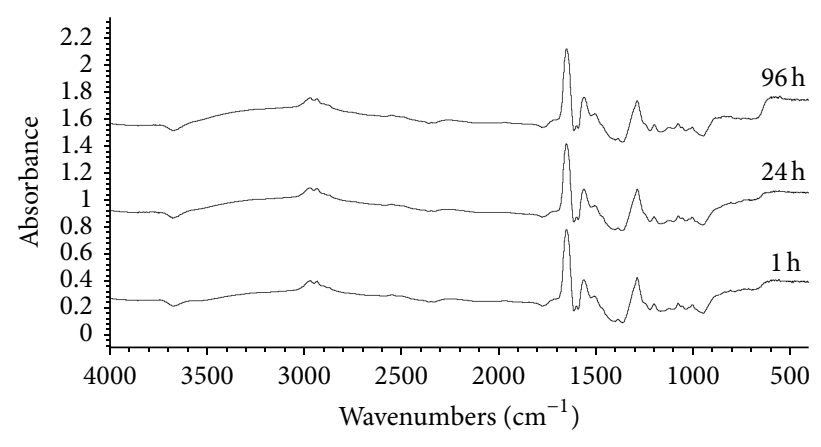

FIgURE 4: Subtracted FTIR spectra of EHAM 1 and 2-CEES.

Table 5 summarizes the relative intensity data for all the products present in the fluid.

Time-dependent ${ }^{1} \mathrm{H}$ NMR spectra of DFP in Gamble's fluid containing EHAMs 1, 2, and 4 are displayed in Figures 11(a)-11(c), respectively. On the basis of peak patterns observed in the $1.0-1.3 \mathrm{ppm}$ region, it is readily 
TABLE 5: Comparison of methyl-peak intensities of DFP reaction products.

\begin{tabular}{|c|c|c|c|c|c|c|c|c|c|}
\hline \multirow{3}{*}{ Peak } & \multicolumn{9}{|c|}{ Relative intensity percentage } \\
\hline & \multicolumn{3}{|c|}{$\mathrm{D}_{2} \mathrm{O}$} & \multicolumn{3}{|c|}{ Saline } & \multicolumn{3}{|c|}{ Gamble's } \\
\hline & $1 \mathrm{~h}$ & $24 \mathrm{~h}$ & $96 \mathrm{~h}$ & $1 \mathrm{~h}$ & $24 \mathrm{~h}$ & $96 \mathrm{~h}$ & $1 \mathrm{~h}$ & $24 \mathrm{~h}$ & $96 \mathrm{~h}$ \\
\hline g & 0.9 & 4 & 5 & 1 & 4 & 4 & 0.7 & 4.4 & 11 \\
\hline $\mathrm{h}$ & 2 & 18 & 5 & 2 & 25 & 0 & 2.2 & 15 & 20 \\
\hline $\mathrm{i}$ & 1 & 3 & 89 & 1 & 4 & 94 & 0.7 & 4.2 & 11 \\
\hline j & 2 & 2 & 1.5 & 2 & 2 & 1 & 1.8 & 1.8 & 1.8 \\
\hline f & 94 & 73 & 0 & 94 & 64 & 0.5 & 94 & 74 & 56 \\
\hline Assignment & \multicolumn{2}{|c|}{ IPA (g) } & \multicolumn{2}{|c|}{ TIPPP (h) } & DIPP (i) & \multicolumn{2}{|c|}{$\operatorname{IPP}(j)$} & \multicolumn{2}{|c|}{ DFP (f) } \\
\hline
\end{tabular}

TABLE 6: Comparison of methyl-peak intensities of DFP reaction products.

\begin{tabular}{|c|c|c|c|c|c|c|c|c|c|c|c|c|}
\hline \multirow{3}{*}{ peak } & \multicolumn{12}{|c|}{ Relative intensity percentage } \\
\hline & \multicolumn{3}{|c|}{ Gamble's } & \multicolumn{3}{|c|}{ EHAM 1} & \multicolumn{3}{|c|}{ EHAM 2} & \multicolumn{3}{|c|}{ EHAM 4} \\
\hline & $1 \mathrm{~h}$ & $24 \mathrm{~h}$ & $96 \mathrm{~h}$ & $1 \mathrm{~h}$ & $24 \mathrm{~h}$ & $96 \mathrm{~h}$ & $1 \mathrm{~h}$ & $24 \mathrm{~h}$ & $96 \mathrm{~h}$ & $1 \mathrm{~h}$ & $24 \mathrm{~h}$ & $96 \mathrm{~h}$ \\
\hline g & 0.7 & 4.4 & 11 & 2 & 15 & 24 & 0.8 & 1.6 & 2 & 6 & 7 & 1 \\
\hline $\mathrm{h}$ & 2.2 & 15 & 20 & 0 & 0 & 0 & 0 & 0 & 0 & 0 & 0 & 0 \\
\hline $\mathrm{i}$ & 0.7 & 4.2 & 11 & 3 & 51 & 65 & 97 & 96 & 96 & 93 & 91 & 96 \\
\hline j & 1.8 & 1.8 & 1.8 & 3 & 11 & 12 & 2 & 2 & 2 & 1 & 1 & 2 \\
\hline $\mathrm{f}$ & 94 & 74 & 56 & 92 & 22 & 0 & 0.8 & 0.8 & 0.9 & 0.5 & 0.7 & 1 \\
\hline Assignment & \multicolumn{2}{|c|}{ IPA $(\mathrm{g})$} & \multicolumn{3}{|c|}{ TIPPP (h) } & \multicolumn{2}{|c|}{ DIPP (i) } & \multicolumn{2}{|c|}{$\operatorname{IPP}(\mathrm{j})$} & \multicolumn{3}{|c|}{$\operatorname{DFP}(f)$} \\
\hline
\end{tabular}

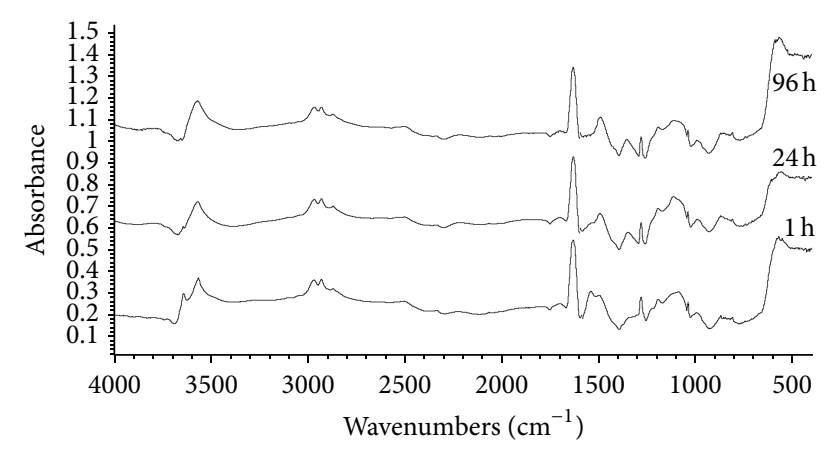

FIGURE 5: Subtracted FTIR spectra of EHAM 2 and 2-CEES.

deduced that EHAMs 2 and 4 were very efficient in degrading DFP. Table 6 displays the related methyl peak intensity data for this set of samples.

3.4.2. Solid State ${ }^{31} P$ NMR Studies. A reference protondecoupled solid state ${ }^{31} \mathrm{P}$ NMR of DFP was collected by adding DFP to low surface area conventional $\mathrm{TiO}_{2}\left(\mathrm{FS}-\mathrm{TiO}_{2}\right.$, $\mathrm{SSA}=11 \mathrm{~m}^{2} / \mathrm{g}$, shown as DFP control in Figure 12). The doublet arises from the scalar coupling between fluorine and phosphorous atoms $\left({ }^{1} J_{\mathrm{PF}}=969 \mathrm{~Hz}\right)$. The nonporous, low surface area $\mathrm{TiO}_{2}$, even though chemically similar to the $\mathrm{TiO}_{2}$ present in the EHAMs, did not interact significantly with DFP. When DFP was allowed to react with EHAM 1, the doublet broadened and began to coalesce (Figure 12(a)), indicating hydrolysis and binding to the surface. With EHAM 2 (Figure 12(b)), the reaction occurred much faster, as was observed by solution ${ }^{1} \mathrm{H}$ NMR studies (Figure $11(\mathrm{~b})$ ). The

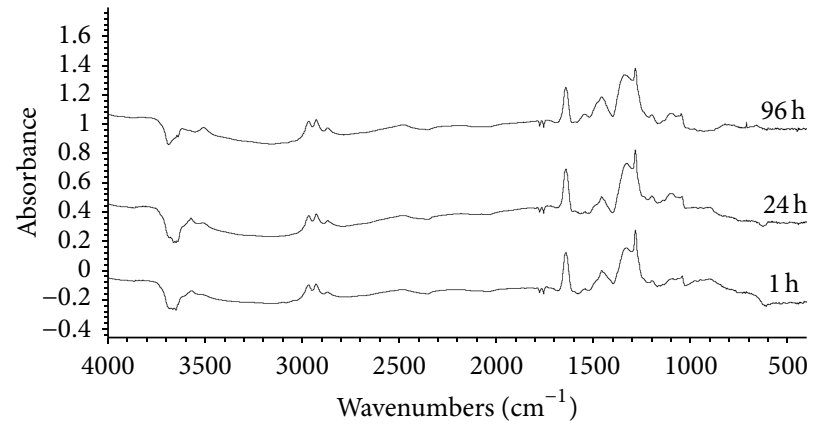

FIGURE 6: Subtracted FTIR spectra of EHAM 4 and 2-CEES.

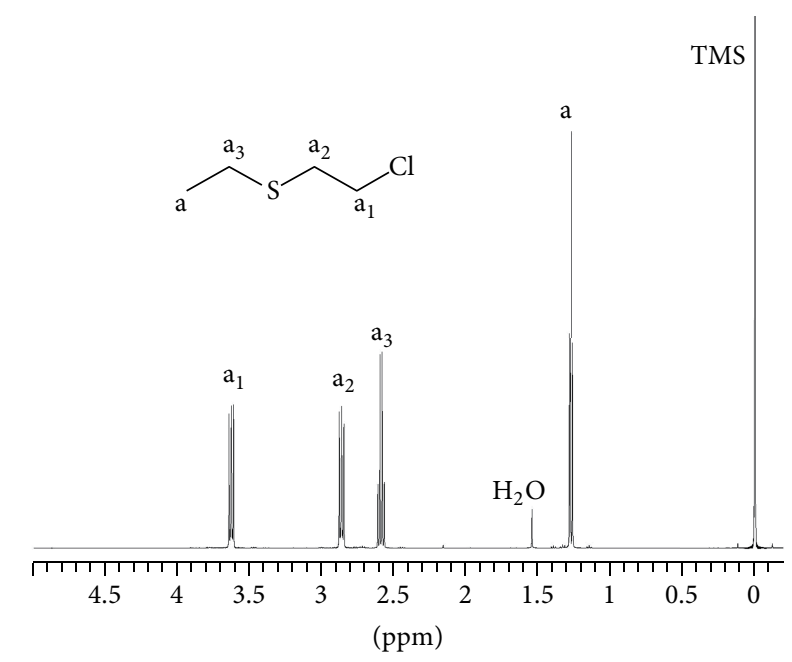

Figure 7: ${ }^{1} \mathrm{H}$ NMR spectrum of 2-CEES in $\mathrm{CDCl}_{3}$. 


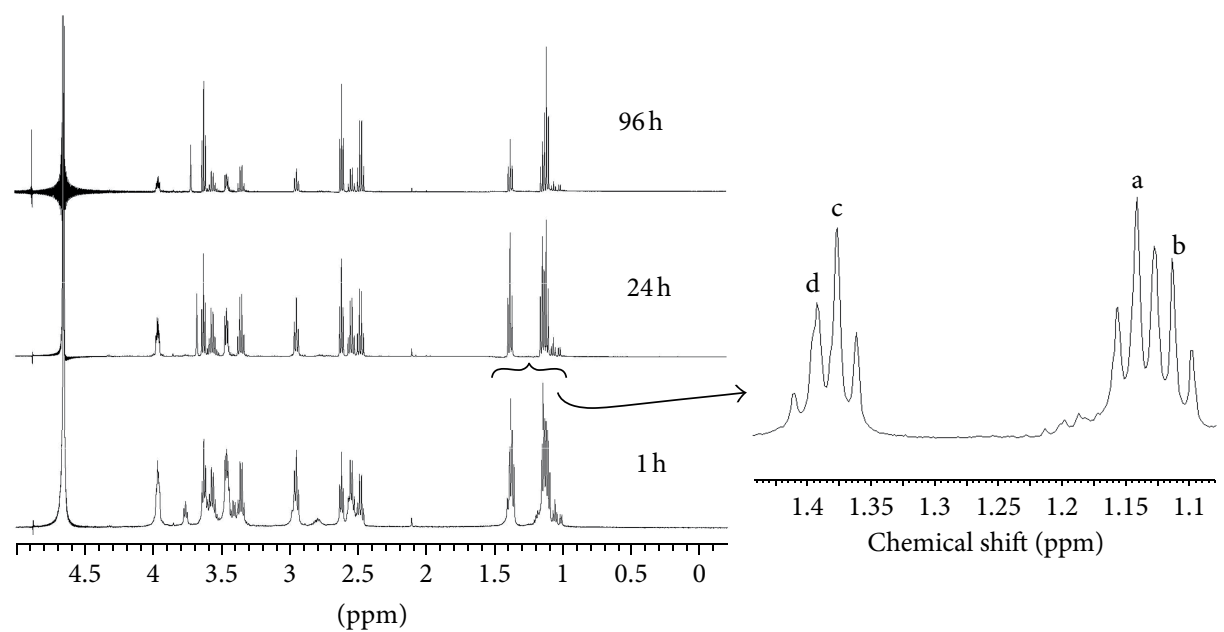

FIGURE 8: ${ }^{1} \mathrm{H}$ NMR spectra of 2-CEES in Gamble's fluid.

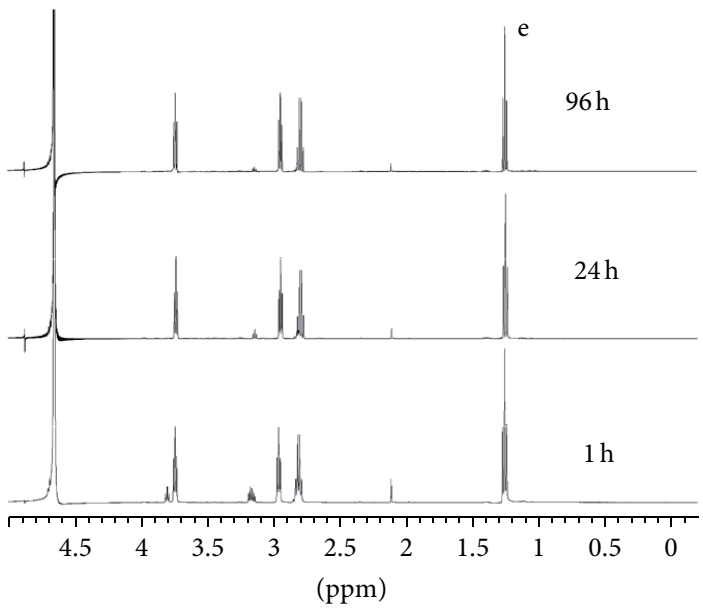

(a)

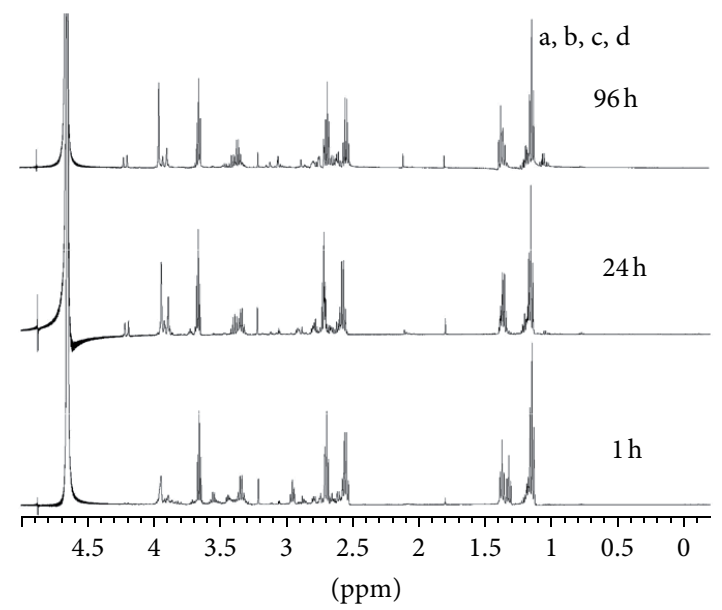

(b)

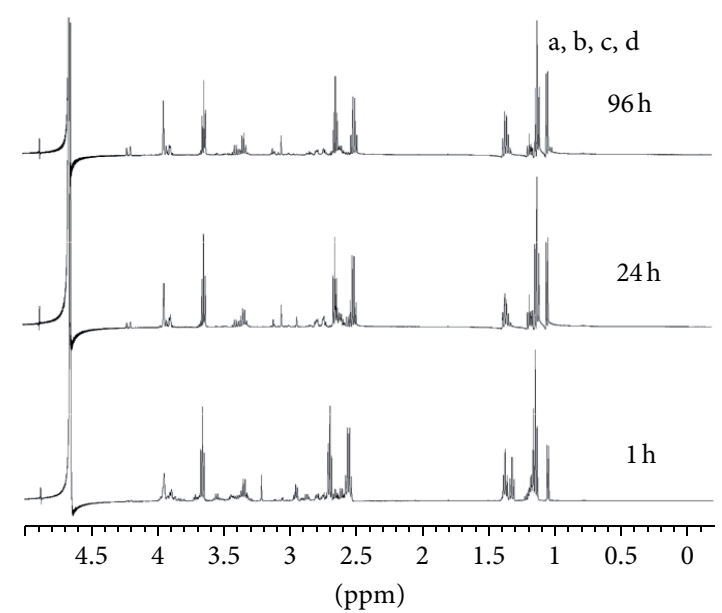

(c)

Figure 9: ${ }^{1}$ H NMR spectra of 2-CEES in Gamble's fluid with (a) EHAM 1, (b) EHAM 2, and (c) EHAM 4. 


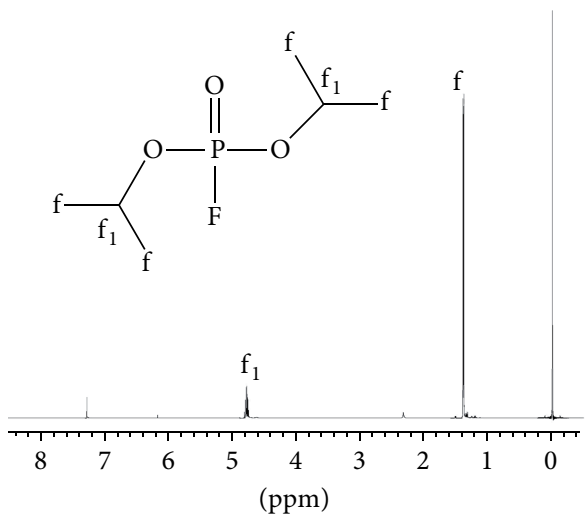

(a)

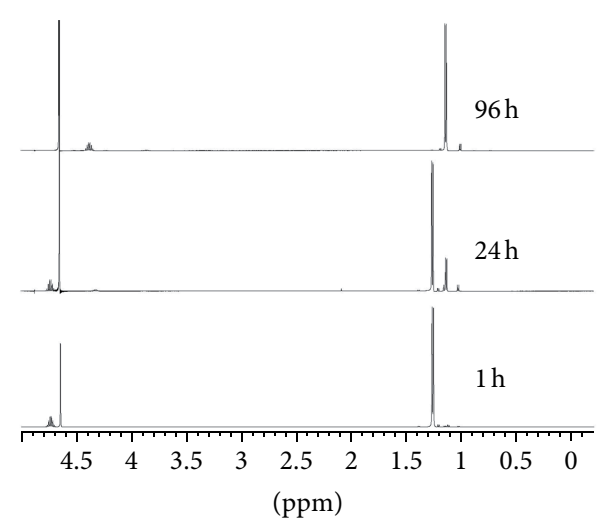

(b)

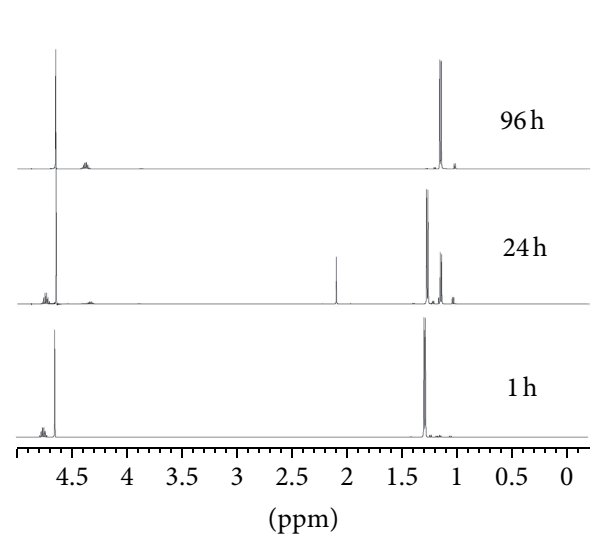

(c)

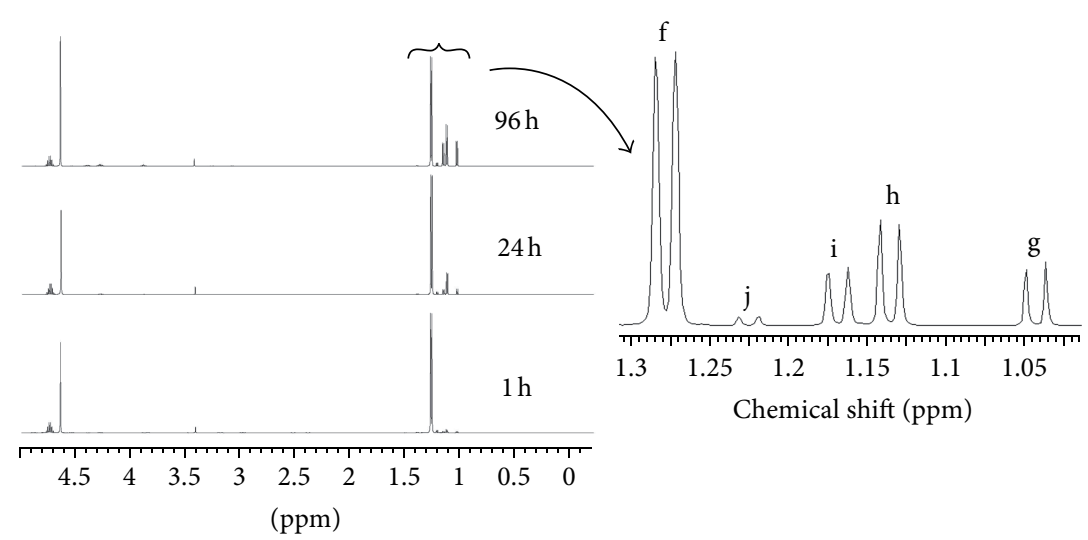

(d)

Figure 10: ${ }^{1} \mathrm{H}$ NMR spectra of DFP in (a) $\mathrm{CDCl}_{3}$, (b) $\mathrm{D}_{2} \mathrm{O}$, (c) saline, and (d) Gamble's fluid.

degradation appears to be complete within $24 \mathrm{~h}$, as indicated by the appearance of a broad peak at $-3.12 \mathrm{ppm}$. Similar results are obtained with EHAM 4 (Figure 12(c)). After $1 \mathrm{~h}$ of reaction, two broad peaks were seen at -5.67 and $-12.9 \mathrm{ppm}$, and within $24 \mathrm{~h}$ only a broad peak at $-4.54 \mathrm{ppm}$ remained. The increased broadness of this peak after $96 \mathrm{~h}$ suggests the presence of multiple phosphorus species in this sample.

\section{Discussion}

4.1. $\mathrm{pH}$ Analysis. Changes in $\mathrm{pH}$ of the simulant-contacted fluids (water, saline, and Gamble's fluid) indicate the occurrence of a reaction or reactions that produce acidic compounds. These changes were observed for both 2-CEES and DFP. Gamble's fluid slows down the rate of drop in $\mathrm{pH}$, likely due to the buffering effect of salts and amino acids present in the solution.

4.2. GC Analysis. As seen from Table 1, the amount of extracted 2-CEES was significantly lower than the control (2-CEES in ethyl ether); however, extracted HEES increased significantly from $1 \mathrm{~h}$ to $96 \mathrm{~h}$ for all the fluids. Only trace levels of condensed (dimer or trimer derived) products were extracted. Similarly, trace levels of DEDS were observed after $1 \mathrm{~h}$ and $24 \mathrm{~h}$ time points for all the fluids, but were not seen by $96 \mathrm{~h}$. This could be due to evaporative loss of DEDS. The origin of DEDS in these samples is not known. A similar trend was observed for EVS as well.

When experiments were performed using EHAMs in Gamble's fluid (Table 2), the amount of recovered 2-CEES was significantly lower when compared to fluids alone (Table 1). The only exception to this was EHAM 1 sample with $1 \mathrm{~h}$ contact time. It is likely that hydrolysis of 2-CEES in the presence of EHAM 1 is much more controlled due to its affinity for water. Interestingly, fluids from EHAMs 2 and 4 contained as much HEES as did Gamble's fluid. In contrast, EHAM 1 fluid had only minor amounts of HEES as the reaction product, which could be attributed to the high adsorptive power of EHAM 1. A minor amount of NEES was observed only with EHAM 1.

As attested by GC analysis (Figure 2), degradation of DFP was significantly faster and essentially complete in water and saline. However, in Gamble' fluid, DFP degradation was only partial (Figure $2(\mathrm{a})$ ). This sharp difference in hydrolytic power of the fluids was also further confirmed by NMR 


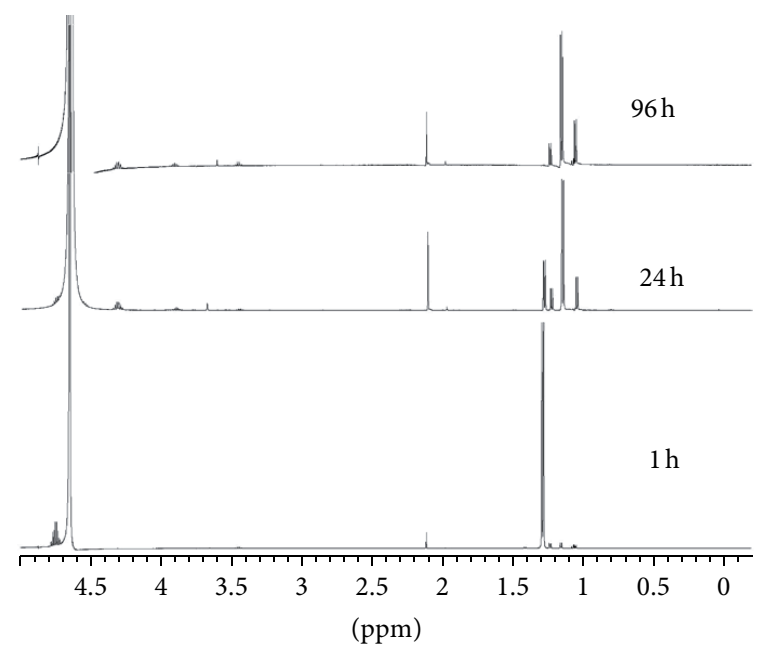

(a)

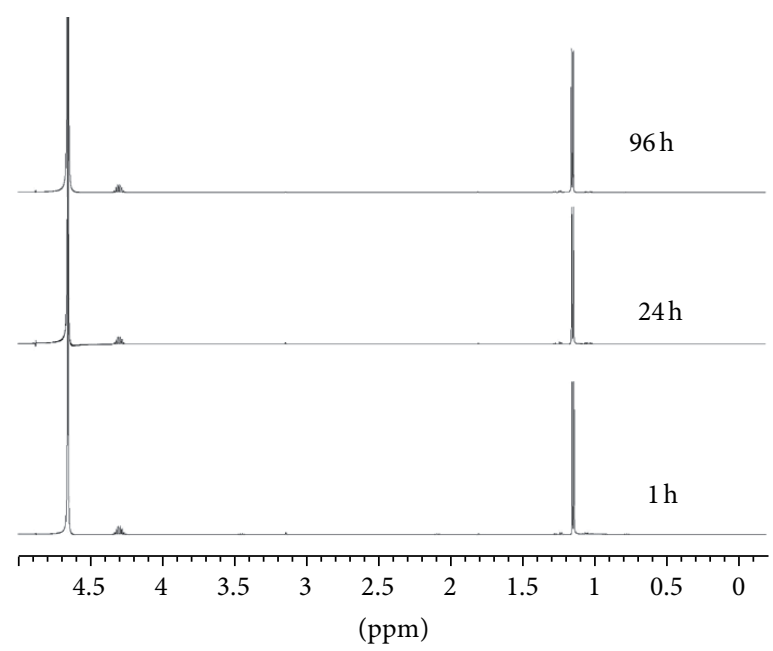

(b)

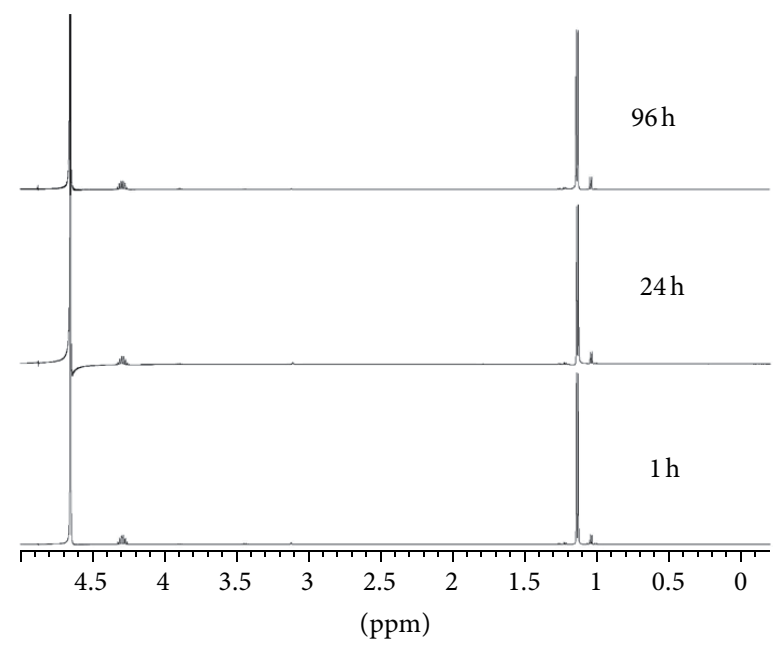

(c)

FIGURE 11: ${ }^{1}$ H NMR spectra of DFP in Gamble's fluid with (a) EHAM 1, (b) EHAM 2, and (c) EHAM 4.

results (Figure 10 and Table 5). In contrast, samples involving DFP exposed to EHAMs in Gamble's fluid had significantly lower amounts of extracted DFP than Gamble's fluid alone (compare Figures 2(a) and 2(b)).

4.3. FTIR Analysis. The negative absorption feature at $\sim 3700 \mathrm{~cm}^{-1}$ from reaction of 2-CEES and EHAM 1 (Figure 4) indicates that hydrogen bonding was involved in the adsorption of 2-CEES by EHAM 1. It was previously reported that the surface $\mathrm{MO}-\mathrm{H}$ groups can readily hydrogen bond to the sulfur and chlorine atoms in 2-CEES [13]. The absence of the $\mathrm{C}-\mathrm{Cl}$ functionality suggested that 2-CEES was consumed; the newly formed bands $\left(1640 \mathrm{~cm}^{-1}, \mathrm{ONO}_{2}\right.$ asymmetric stretch; $1280 \mathrm{~cm}^{-1}, \mathrm{ONO}_{2}$ symmetric stretch) were attributable to the NEES product [14]. In contrast, EHAMs 2 and 4 display a positive hydroxyl band attesting to the formation of significant amounts of hydroxyl product.
All the dry EHAM formulations show the presence of NEES as one of the degradation products of 2-CEES.

\subsection{NMR Analysis}

4.4.1. Solution ${ }^{1}$ H NMR Studies. 2-CEES undergoes hydrolysis to yield at least three products after $96 \mathrm{~h}$. One of the products is identified as HEES, while the other two products are likely to be the two sulfonium condensation products (dimer and/or trimer in Scheme 2) [15]. These conclusions are supported by GC-MS data. It is difficult to differentiate between the various condensed products ( $\mathrm{R}-\mathrm{Cl}$ and $\mathrm{R}-\mathrm{OH}$ or dimer versus trimer) by NMR because their structures are highly similar with chemically equivalent hydrogens.

Very similar changes are observed for 2-CEES in all the three fluids (Table 3). This reinforces the inference drawn that the slower $\mathrm{pH}$ drop in Gamble's fluid is due to its buffering capacity, rather than a reduced rate of hydrolysis 


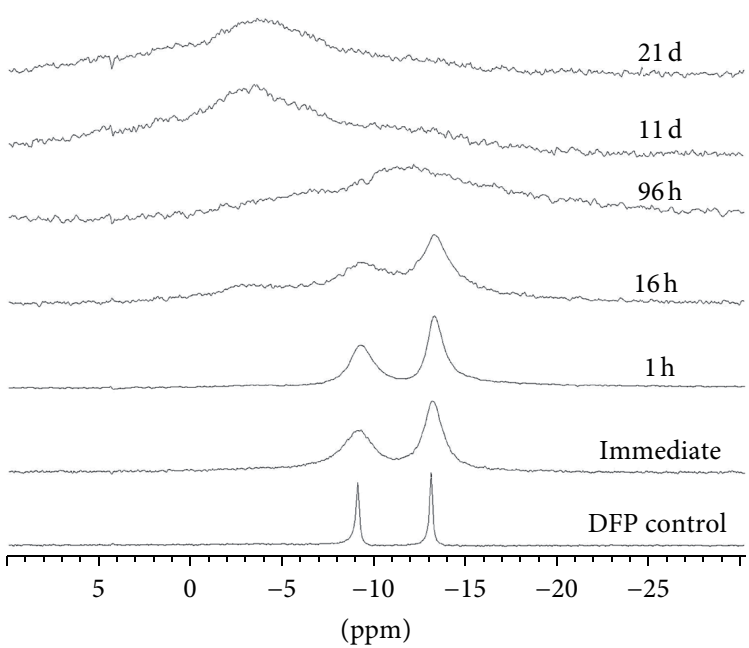

(a)

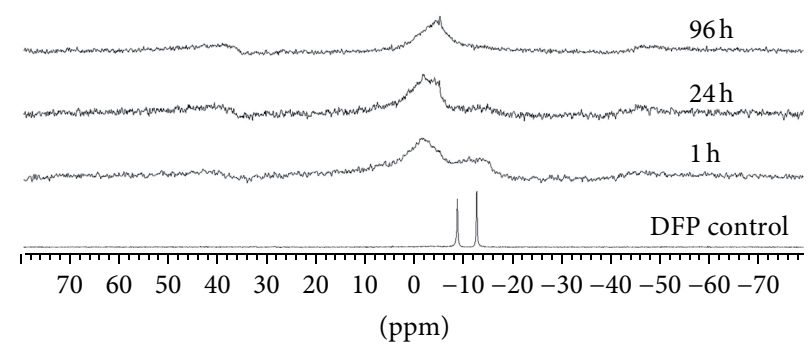

(b)

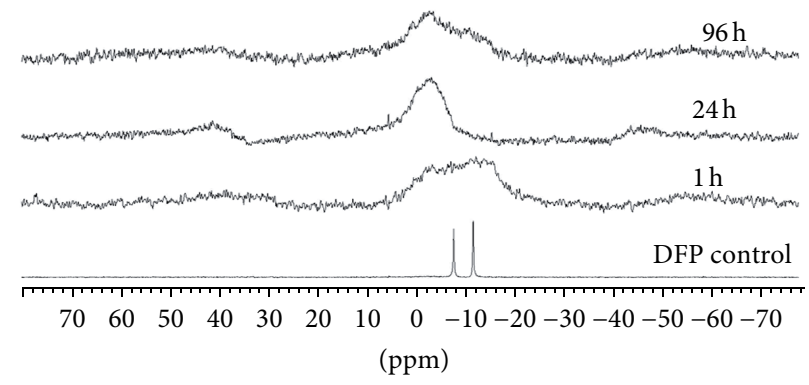

(c)

FIgURE 12: ${ }^{31}$ P MAS NMR spectra of DFP with (a) EHAM 1, (b) EHAM 2, and (c) EHAM 4.

and degradation of the simulant in that solution. In $\mathrm{D}_{2} \mathrm{O}$ and saline, after $96 \mathrm{~h}$, roughly equal amounts of 2-CEES, HEES, and the condensed products result, whereas in Gamble's fluid, HEES is formed as a major product.

Differences in the amounts of reactions products formed, as monitored by the two methods, may arise out of degradation of the condensed products, back into 2-CEES and HEES on the GC column [15].

Interestingly, EHAM 1-contacted Gamble's fluid contained only one product, namely, NEES. Additional products might have formed and been trapped on the sorbent. EHAM 2 and EHAM 4 showed similar degradation results with the amount of HEES formed being around 50\% after $96 \mathrm{~h}$. However, in both cases, higher levels of the condensed products were observed after $96 \mathrm{~h}$, as compared to 2-CEES in Gamble's fluid or EHAM 1. The NMR results are consistent with the GC-MS data, especially those related to extractable amounts of HEES from all the EHAMs (Tables 2 and 4). Overall, it is concluded that EHAMs accelerate the degradation of 2-CEES with EHAM 1 being the most efficient in keeping the fluid less toxic.

In the case of DFP, analysis of its degradation products in $\mathrm{D}_{2} \mathrm{O}$, saline, and Gamble's fluid, as deduced from relative intensities of various peaks (Table 5), suggests that DFP undergoes loss of fluorine to yield DIPP, which is subsequently degraded to form IPA and IPP as minor products [16]. The hydrolysis rate is the slowest in Gamble's fluid with DFP as a major component of the products observed even after $96 \mathrm{~h}$. The solution ${ }^{1} \mathrm{H}$ NMR results of DFP in fluids are consistent with the GC-MS data (Figure 2).

The relative intensities of various methyl peaks in the spectra of DFP and EHAMs are shown in Table 6. The most notable result is that complete degradation of DFP is readily achieved by EHAM inclusion. As with DFP in fluids, the amount of DFP is decreased and the major product is DIPP (Peak i). EHAM 1 reacts with DFP slowly since 92\% of the methyl peaks are from DFP after $1 \mathrm{~h}$, while with EHAMs 2 and 4 , less than $1 \%$ is seen for the same time period. However, after 96 h, DFP has been degraded nearly completely by all of the EHAMs. EHAMs 2 and 4 are very quick to destroy DFP with almost the entire product formed being the monohydrolyzed product (DIPP). It appears that EHAM 1 further hydrolyzes DFP to IPP, as seen by the higher levels of IPA (Peak g) and IPP (Peak j). The results in the presence of EHAMs also match very closely for both GC and NMR analysis. In both experiments, the level of DFP decreases to $0 \%$ after $96 \mathrm{~h}$ for EHAM 1, but for EHAMs 2 and 4, the level drops to $0 \%$ within $1 \mathrm{~h}$. 
4.4.2. Solid State NMR. Solid state ${ }^{31} \mathrm{P}$ NMR spectra of DFP treated with EHAM 1 are consistent with the formation of hydrolysis products as observed in solution ${ }^{1} \mathrm{H}$ NMR spectra. The initial spectrum of the DFP control shows a very sharp doublet and does not appear to be bound to the low surface area $\mathrm{TiO}_{2}$ support. Broadening of the two peaks after $1 \mathrm{~h}$ suggests binding of the compound to the EHAM surface. After $16 \mathrm{~h}$, the peaks broaden further and begin to merge into a broad band at $-10.8 \mathrm{ppm}$, thus indicating the cleavage of fluorine atom to yield DIPP [17]. In addition, a new broad peak appears at $-3.15 \mathrm{ppm}$ due to further hydrolysis of DIPP to IPP. IPP has a similar structure to phosphoric acid. This is consistent with the solution ${ }^{1} \mathrm{H}$ NMR data when DFP is treated with EHAM 1 in Gamble's fluid (Table 6). By $96 \mathrm{~h}$, the hydrolysis reaction is complete to form DIPP $(-10.8 \mathrm{ppm})$, which is further hydrolyzed to IPP $(-3.59 \mathrm{ppm})$. Between $96 \mathrm{~h}$ and $11 \mathrm{~d}$, the hydrolysis reaction is complete, as no further changes are noted up to $21 \mathrm{~d}$.

Reactions of DFP with EHAMs 2 and 4 yielded similar results to EHAM 1, except that the reactions occurred much faster, as noted from solution ${ }^{1} \mathrm{H}$ NMR studies. Slight variations in chemical shifts are expected for the reaction products based on different metal oxide surfaces [18].

\section{Conclusions}

$\mathrm{pH}$ measurement of fluids is a good indicator of CWA simulant degradation, since acid by-products result from hydrolysis of these compounds. Similarly, solvent extraction of CWA simulant-contacted fluids and subsequent GC analysis provide an overview of various degradation products. For the safe handling of contaminated human remains, it is necessary to use pouches enhanced with EHAMs because contaminated body fluids are expected to contain appreciable amounts of intact agent (as seen by 2-CEES and DFP analysis) or their derivatives (2-CEES analysis). NMR analysis of simulantcontacted fluids revealed that the rate of degradation is highly fluid dependent for DFP (Gamble's $<\mathrm{D}_{2} \mathrm{O} \sim$ saline) and 2-CEES (Gamble's $>\mathrm{D}_{2} \mathrm{O} \sim$ saline). Inclusion of EHAMs facilitates degradation of both DFP and 2-CEES in these fluids, especially during the early hours of contamination. The toxicity of the contaminated Gamble's fluid can be minimized by including an appropriately formulated EHAM sorbent. The ongoing threat of homicidal use of CWAs during military conflicts and by terrorists underlines the necessity for development of clear guidelines that will guarantee foolproof protection of personnel dealing with victims of these serious incidents. Early recognition and protective measures are essential when dealing with CWA-related incidents, otherwise, responding human remains handlers will only add to the list of victims.

\section{Abbreviations}

BEE: 1, 2-bis(ethylthio) ethane

BWAs: Biological Warfare Agents

$\mathrm{CDCl}_{3}$ : Deuterated choroform

CHRP: Contaminated human remain pouch

CONUS: Continental United States
CWAs: Chemical Warfare Agents

DEDS: Diethyl disulfide

DTRA: Defense Threat Reduction Agency

DFP: Diisopropyl fluoro phosphonate

DIPP: Diisopropyl phosphoric acid

DRIFTS: Diffuse Reflectance Infrared Fourier

Transform Spectroscopy

EHAMs: Exceptional Hazard Attenuation Materials

EVS: Ethyl vinyl sulfide

FTIR: $\quad$ Fourier Transform Infrared

FS- $\mathrm{TiO}_{2}$ : Fisher Scientific titanium (IV) oxide

GC: $\quad$ Gas Chromatography

GD: Soman

HD: $\quad$ Mustard

HEES: Hydroxyl ethyl ethyl sulfide

IED: Improvised Explosive Device

IPA: Isopropyl alcohol

IPP: $\quad$ Isopropyl phosphoric acid

MADCP: Mortuary Affairs Decontamination

Collection Point

MAS: $\quad$ Magic Angle Spinning

NA-CaO: NanoActive $\mathrm{CaO}$

NA-TiO 2 : NanoActive $\mathrm{TiO}_{2}$

NEES: $\quad$ 2-Nitroethyl ethyl sulfide

PA: $\quad$ Phosphoric acid

SSA: $\quad$ Specific surface area

TIPPP: Tetra isopropoxy pyrophosphate

NMR: Nuclear Magnetic Resonance

TICs: $\quad$ Toxic Industrial Chemicals

USARO: US Army Research Office

2-CEES: 2-Chloro ethyl ethyl sulfide.

\section{Conflict of Interests}

The authors report no conflict of interests.

\section{Acknowledgments}

This paper was funded by the Defense Threat Reduction Agency (DTRA) and US Army Research Office (USARO) W911NF-09-C-0116. The authors thank Mr. Alvaro Herrera (Biomolecular NMR Facility, Kansas State University) and $\mathrm{Dr}$. Shu Xu (Magnetic Resonance Facility, Iowa State University) for assistance in acquiring NMR data.

\section{References}

[1] R. A. Falkenrath, R. D. Newman, and B. A. Thayer, America's Achilles' Heel: Nuclear, Biological, and Chemical Terrorism and Covert Attack, The MIT Press, Cambridge, Mass, USA, 1998.

[2] D. A. Shea and F. Gottron, "Small-scale terrorist attacks using chemical and biological agents: an assesment framework and preliminary comparisons," CRS Report For Congress RL32391, 2004.

[3] A. T. Tu, "Toxicological and chemical aspects of sarin terrorism in Japan in 1994 and 1995," Toxin Reviews, vol. 26, no. 3, pp. 231-274, 2007.

[4] J. M. Stewart and U. S. Army, Mortuary Affairs-Is USNORTHCOM and the Department of Homeland Security Positioned for 
Contaminated Mass Fatality Management?United States Army War College, 2004.

[5] B. Papirmeister, R. Ford, S. Robinson et al., Issues Related to the Decontamination of Chemically Contaminated Human Remains. United States Army Medical Research Institute of Chemical Defense, USAMRICD-TR-93-02, 1993.

[6] D. Poyer, Handling of Human Remains from Natural Disasters. U.S. Army Center for Health Promotion and Preventative Medicine, 2005.

[7] A. M. Iseli, H. D. Kwen, M. Ul-Alam, M. Balasubramanian, and S. Rajagopalan, "Enhanced Contaminated Human Remains Pouch: initial development and preliminary performance assessments," American Journal of Disaster Medicine, vol. 6, no. 1, pp. 31-38, 2011.

[8] S. Rajagopalan, O. Koper, S. Decker, and K. J. Klabunde, "Nanocrystalline metal oxides as destructive adsorbents for organophosphorus compounds at ambient temperatures," Chemistry - A European Journal, vol. 8, no. 11, pp. 2602-2607, 2002.

[9] G. W. Wagner, P. W. Bartram, O. Koper, and K. J. Klabunde, "Reactions of VX, GD, and HD with nanosize MgO," Journal of Physical Chemistry B, vol. 103, no. 16, pp. 3225-3228, 1999.

[10] G. W. Wagner, O. B. Koper, E. Lucas, S. Decker, and K. J. Klabunde, "Reactions of VX, GD, and HD with nanosize CaO: autocatalytic dehydrohalogenation of HD," Journal of Physical Chemistry B, vol. 104, no. 21, pp. 5118-5123, 2000.

[11] G. W. Wagner, L. R. Procell, R. J. O'Connor et al., "Reactions of $\mathrm{VX}, \mathrm{GB}, \mathrm{GD}$, and $\mathrm{HD}$ with nanosize $\mathrm{AL}_{2} \mathrm{O}_{3}$. Formation of aluminophosphonates," Journal of the American Chemical Society, vol. 123, no. 8, pp. 1636-1644, 2001.

[12] J. Valentin, "Guide for the practical application of the ICRP Human Respiratory Tract Model. A report of ICRP supporting guidance 3: approved by ICRP committee 2 in October 2000," Annals of the ICRP, vol. 32, no. 1-2, pp. 13-306, 2002.

[13] D. Panayotov and J. T. Yates, "Bifunctional hydrogen bonding of 2-chloroethyl ethyl sulfide on $\mathrm{TiO}_{2}-\mathrm{SiO}_{2}$ powders," Journal of Physical Chemistry B, vol. 107, no. 38, pp. 10560-10564, 2003.

[14] E. A. Bruns, V. Perraud, A. Zelenyuk et al., "Comparison of FTIR and particle mass spectrometry for the measurement of particulate organic nitrates," Environmental Science and Technology, vol. 44, no. 3, pp. 1056-1061, 2010.

[15] Y. C. Yang, L. L. Szafraniec, W. T. Beaudry, and J. R. Ward, "Kinetics and mechanism of the hydrolysis of 2-chloroethyl sulfides," Journal of Organic Chemistry, vol. 53, no. 14, pp. 3293-3297, 1988.

[16] Y. C. Yang, J. A. Baker, and J. R. Ward, "Decontamination of chemical warfare agents," Chemical Reviews, vol. 92, no. 8, pp. 1729-1743, 1992.

[17] G. W. Wagner and P. W. Bartram, "Reactions of the nerve agent simulant diisopropyl fluorophosphate with selfdecontaminating adsorbents. A ${ }^{31}$ P MAS NMR study," Journal of Molecular Catalysis A, vol. 144, no. 3, pp. 419-424, 1999.

[18] G. W. Wagner and R. A. Fry, "Observation of distinct surface AlIV sites and phosphonate binding modes in $\gamma$-alumina and concrete by high-field ${ }^{27} \mathrm{Al}$ and ${ }^{31} \mathrm{P}$ MAS NMR," Journal of Physical Chemistry C, vol. 113, no. 30, pp. 13352-13357, 2009. 

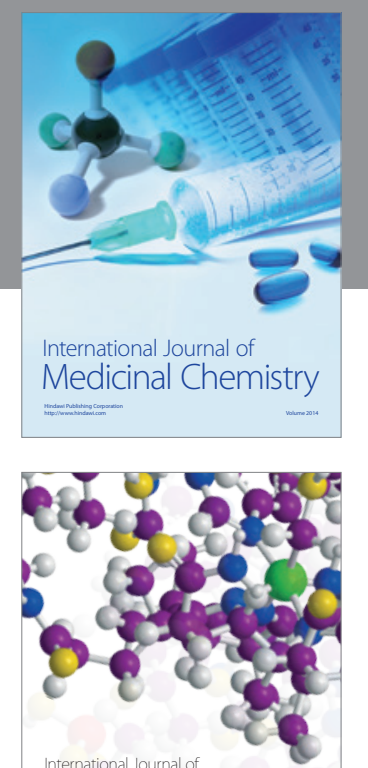

\section{Carbohydrate} Chemistry

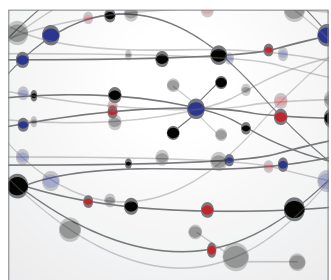

The Scientific World Journal
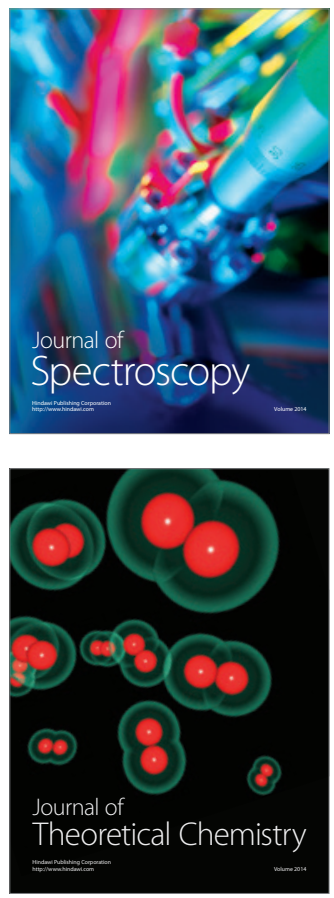
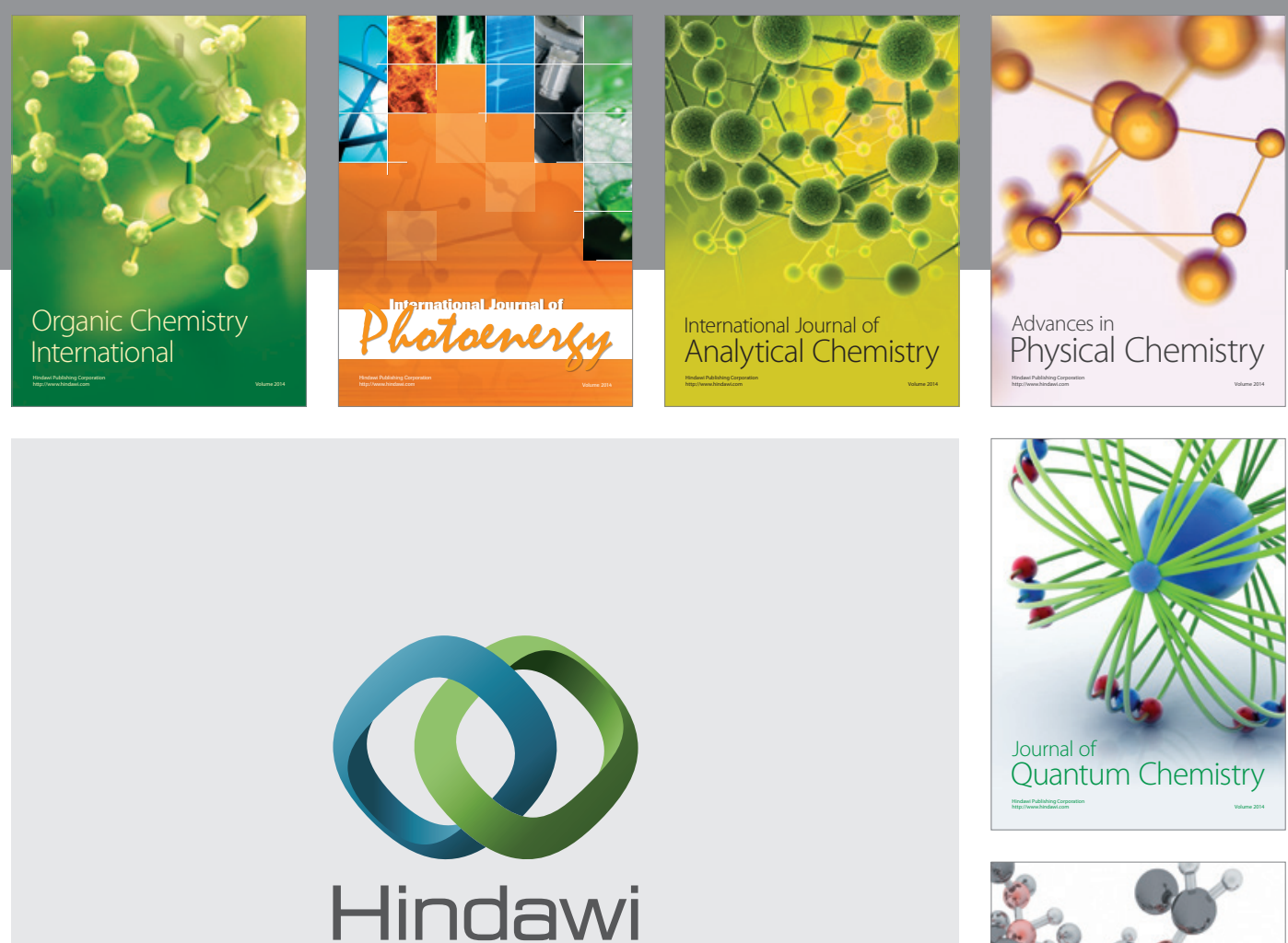

Submit your manuscripts at

http://www.hindawi.com

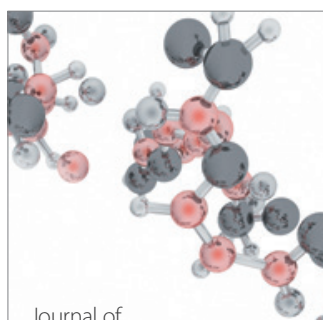

Analytical Methods

in Chemistry

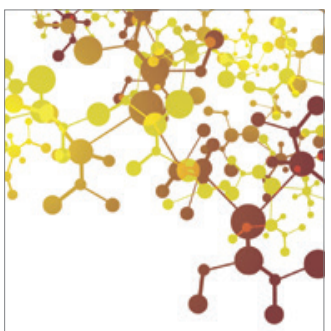

Journal of

Applied Chemistry

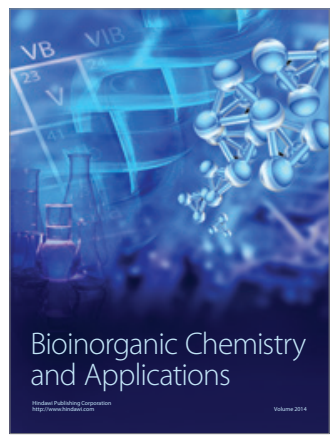

Inorganic Chemistry
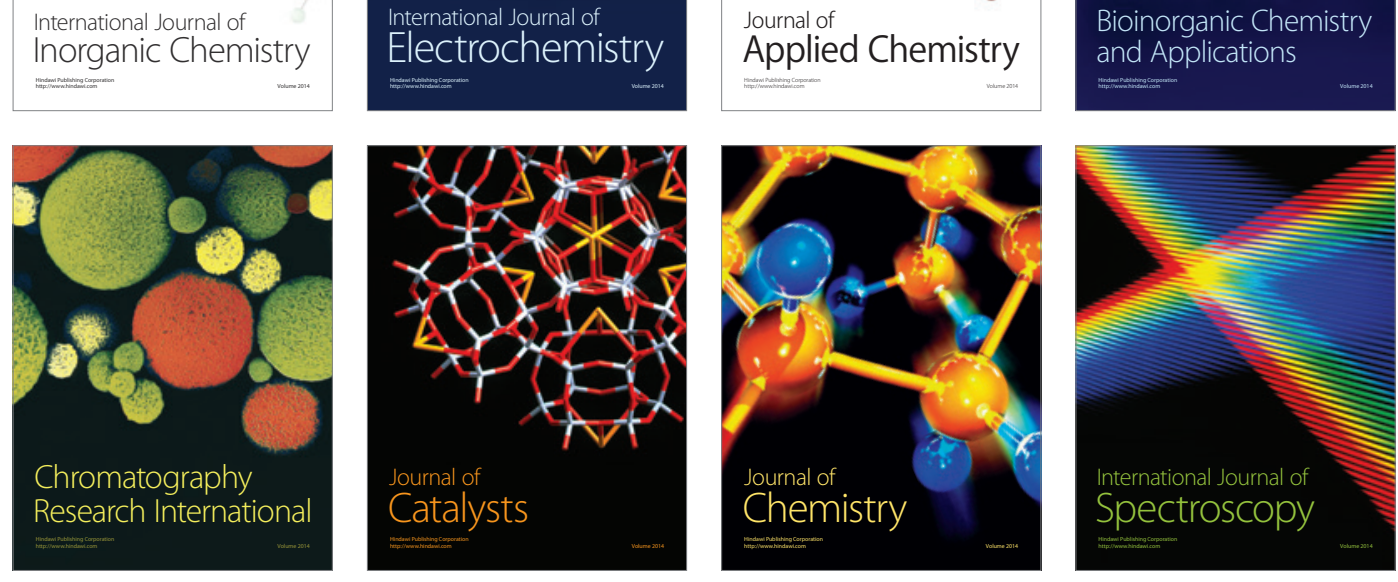